\title{
Determinación de los parámetros reológicos de un champú con el modelo de Carreu-Yasuda
}

Determination of rheological parameters of shampoo with the Carreu-Yasuda model

Recibido: julio 10 de 2015 | Revisado: setiembre 18 de 2015 | Aceptado: octubre 15 de 2015

\author{
Luis Carrasco Venegas ${ }^{\mathrm{I}}$ \\ Luz Castañeda Pérez ${ }^{2}$ \\ Karina Altamirano OnCOY ${ }^{3}$
}

1 Facultad de Ingeniería Química de la Universidad Nacional del Callao clave012@yahoo.es

2 Facultad de Ciencias Naturales de la Universidad Nacional Federico Villarreal lcastaneda@unfv.edu.pe

3 Facultad de Ingeniería Química de la Universidad Nacional del Callao

\begin{abstract}
In this investigation, the calculation of the rheological parameters of a shampoo sample using the Carreu - Yasuda model was performed using the non-linear regression method, which consists in formulating an objective function that is to be the sum of the squared difference between the experimentally-observed data and estimated obtained from the model proposed by Carreu-Yasuda. The objective function is partially derived for each of the five model parameters and then is equaled to zero. This procedure allows us to get five non-linear algebraic equations, which have been resolved using the Polymath software. The experimental data were taken from a publication of the Latin American Journal of Physics. In that article, the experimental data were obtained with the Couette viscometer and were correlated with a commercial software. A calculation software was used as well and similar results to those proposed by Méndez-Sánchez were achieved.
\end{abstract}

Key words: viscosity, non Newtonian fluids, nonlinear regression

\section{Resumen}

En esta investigación, se realizó el cálculo de los parámetros reológicos de una muestra de champú usando el modelo de Carreu - Yasuda, el mismo que se efectuó mediante el método de regresión no lineal, el cual consiste en la formulación de una función objetivo que viene a ser la suma de la diferencia al cuadrado entre los datos observados experimentalmente y los estimados, obtenidos a partir del modelo propuesto mediante el modelo de Carreu-Yasuda. Dicha función objetivo se deriva, parcialmente, respecto a cada uno de los cinco parámetros del modelo y luego se iguala a cero. Este procedimiento permite obtener cinco ecuaciones algebraicas no lineales, que han sido resueltas con el software Polymath. Los datos experimentales fueron tomados de una publicación de la Revista Latinoamericana de Física. En dicho artículo, los datos experimentales se obtuvieron con el viscosímetro de Couette y fueron correlacionados con un software comercial. También se hizo uso de un software de cálculo y se lograron resultados similares al propuesto por Méndez -Sánchez.

Palabras clave: viscosidad, fluidos no newtonianos, regresión no lineal 


\section{Introducción}

Los fluidos no newtonianos presentan ciertas propiedades físicas muy particulares por ello los datos experimentales del estudio observados, deben tomarse en cuenta en cada grupo de estos fluidos.

Es así como, en la actualidad, existen alrededor de 40 modelos de viscosidad de los fluidos, cuyas propiedades son independientes del tiempo. Basado en experiencias de algunos trabajos experimentales, es posible elegir uno o más modelos que caractericen a cierto tipo de fluido no newtoniano.

El comportamiento viscoso de los fluidos no newtonianos es bastante complejo, por ejemplo, se pueden encontrar fluidos cuya viscosidad a valores de velocidad de deformación relativamente bajos, en algunos casos $(\dot{\gamma} \leq 1)$, puede considerarse constante e independiente de la rapidez de deformación, es decir, muestra un comportamiento newtoniano. Para valores de rapidez de corte intermedio $(\leq)$, presentan un comportamiento altamente no newtoniano caracterizado por la ley de potencia (o algún otro modelo). Para valores de deformación relativamente altos $(1<<\dot{\gamma})$, el comportamiento vuelve a ser newtoniano (Méndez-Sánchez, Pérez y Paniagua, 2010).

Precisamente, el champú es un fluido no newtoniano, cuya caracterización reológica es posible realizar mediante el modelo de viscosidad de Carreu-Yasuda.

\section{FUNDAMENTO TEÓRICO}

\section{Fluidos newtonianos}

Un fluido newtoniano es aquel cuya relación entre la velocidad de corte (velocidad de cizallamiento: ) y el esfuerzo de corte (esfuerzo cortante o densidad de flujo viscoso de transporte de cantidad de movimiento); $\tau$, es constante, tal como se observa en la Figura 1.

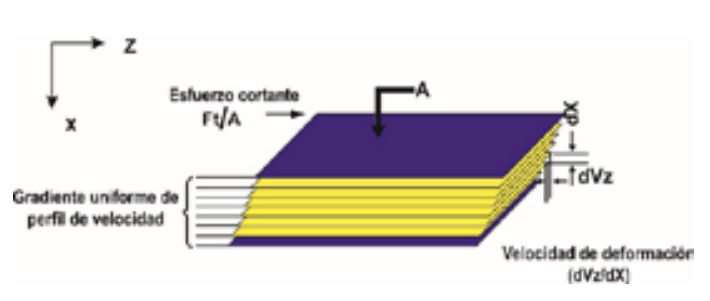

Figura 1. Esquema representativo del esfuerzo de corte $(\tau)$ y la velocidad de corte $\left(\frac{\partial V_{z}}{\partial x}\right)$ para un fluido Newtoniano.

En la Figura 1, la constante de proporcionalidad entre el esfuerzo de corte y la velocidad de corte se denomina viscosidad. A todos los fluidos que obedecen a esta tendencia se les denomina fluidos newtonianos (Carrasco, 2011), cuya relación matemática es:

$$
\tau=\frac{F}{A}=-\mu \frac{\partial V_{z}}{\partial x}=\mu \dot{\gamma}
$$

\section{Fluidos no newtonianos}

Existen algunas sustancias industrialmente importantes que no se comportan siguiendo la ley de Newton de la viscosidad, ya que esta a una temperatura y presión dada es función del gradiente de velocidad o velocidad de deformación. Los fluidos cuya relación entre esfuerzo de corte y velocidad de deformación no es proporcional reciben el nombre de fluidos no-newtonianos.

La mecánica de los fluidos se ocupa del estudio de los fluidos newtonianos exclusivamente mientras que los fluidos no-newtonianos son parte de una ciencia más amplia, denominada Reología.

La Reología es la ciencia que estudia y analiza los fenómenos de flujo y deformación y las propiedades mecánicas de los gases, líquidos, plásticos y comprende el estudio de las substancias que "fluyen", pero que su comportamiento no está regido por la ecua- 
ción 1. Consecuentemente, se puede decir que el campo de la Reología se extiende desde la Mecánica de los Fluidos Newtonianos hasta la elasticidad de Hooke. La región comprendida entre ellas corresponde a todos los materiales pastosos y a las suspensiones (Carrillo, 2011).

En el mundo real, existe una amplia variedad de fluidos tan comunes como los newtonianos que no siguen la simple relación dada por la ley de Newton, especialmente en las industrias químicas, alimenticias y en la industria del petróleo, y de allí la importancia de su estudio para un adecuado y correcto tratamiento. Pueden mencionarse, entre otros, los siguientes fluidos no-newtonianos:

- Pinturas y barnices

- Soluciones de polímeros

- Mermeladas y jaleas

- Mayonesa y manteca

- Dulce de leche y miel

- Salsas y melazas

- Soluciones de agua con arcillas y carbón

- La sangre humana

\section{Características y clasificación de los fluidos no-newtonianos}

Los fluidos que no siguen la relación de proporcionalidad entre tensiones tangenciales y velocidades de deformación se clasifican en tres grupos:

- Fluidos no-newtonianos independientes del tiempo para los cuales se verifica:

$$
\tau=f(\dot{\gamma})
$$

- Fluidos no-newtonianos dependientes del tiempo, en los que la relación anterior es más compleja y puede expresarse como:

$$
\tau=f(\dot{\gamma}, t, \text { historia })
$$

- Fluidos visco-elásticos, fluidos en los que a diferencia de los viscosos donde la energía de deformación es disipada totalmente. Esa energía puede recuperarse como sucede en los sólidos elásticos.

\section{FLUIDOS NO NEWTONIANOS IN- DEPENDIENTES DEL TIEMPO}

Los fluidos no newtonianos independientes del tiempo se caracterizan porque las tensiones tangenciales dependen únicamente de la velocidad de deformación y se representan funcionalmente en tres formas equivalentes:

La gran mayoría de fluidos que tienen aplicaciones en ingeniería, caen dentro de esta categoría, y en algunos casos, algunos fluidos dependientes del tiempo pueden ser aproximados o modelados como fluidos independientes del tiempo.

Para visualizar y analizar los fluidos no newtonianos resulta más cómodo representar el comportamiento de la ecuación 1 en función de un sistema de coordenadas $\tau-\dot{\gamma}$ según indica la Figura 2. Se pueden identificar cinco tipos de fluidos no newtonianos independientes del tiempo.

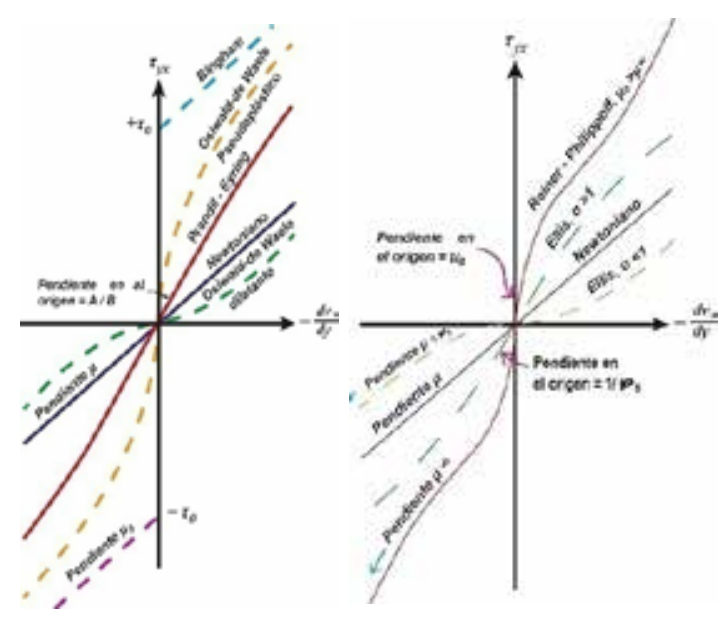




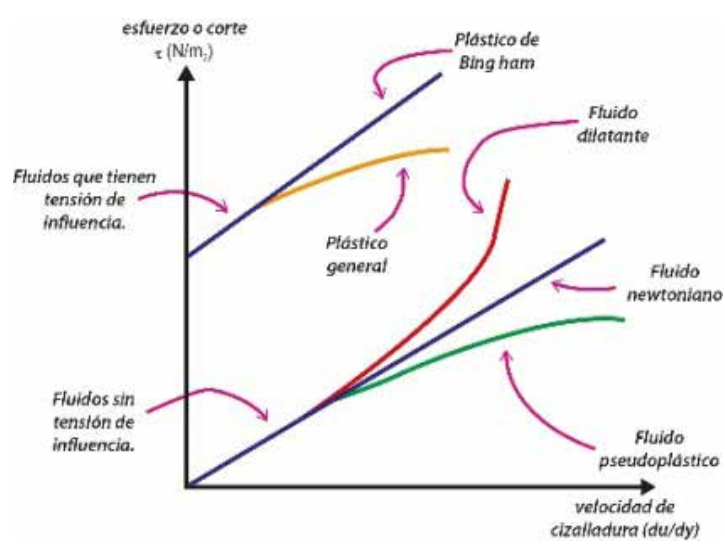

Figura 2: Clasificación de los fluidos no newtonianos según: $\tau \quad(\gamma)$

\section{El modelo de Carreu-Yasuda}

El modelo de Carreu-Yasuda es uno de los modelos independientes de tiempo que contiene cinco parámetros, tal como se indica a continuación:

El modelo de Carreu-Yasuda está dado por la siguiente ecuación:

$\eta=\left(\eta_{0}-\eta_{\infty}\right)\left[1+(\lambda \gamma)^{a}\right]^{\frac{n-1}{a}}+\eta_{\infty}$

Este modelo consta de cinco parámetros: $\eta_{0}, \eta_{\infty}, \lambda, a, n$

$\eta_{0}$ : corresponde a la viscosidad newtoniana a valores de rapidez de deformación bajos.

$\eta_{\infty}$ : es la viscosidad newtoniana para valores de rapidez de deformación altos.

$\lambda$ : es una constante de tiempo.

$n$ : corresponde al parámetro del modelo de ley de potencias.

$a$ : es una constante adimensional.

\section{Método}

\section{Procedimiento de cálculo}

Se disponen de datos de viscosidad aparente y velocidad de cizallamiento para una muestra de champú, un fluido no newtoniano y que obedece al modelo de Carreu-Yasuda (Tabla 1).
Tabla 1

Datos de viscosidad aparente y velocidad de corte para una muestra de champú

\begin{tabular}{|c|c|}
\hline$\eta$ (Pa.s) & $\gamma\left(s^{\wedge}(-1)\right)$ \\
\hline 37.211 & 0.624 \\
\hline 38.771 & 0.700 \\
\hline 39.229 & 0.792 \\
\hline 42.156 & 0.830 \\
\hline 36.623 & 1.17 \\
\hline 37.198 & 1.31 \\
\hline 36.747 & 1.70 \\
\hline 34.592 & 2.26 \\
\hline 33.31 & 2.70 \\
\hline 28.02 & 3.77 \\
\hline 23.77 & 4.61 \\
\hline 12.72 & 8.92 \\
\hline 5.23 & 23.2 \\
\hline 5.07 & 24.7 \\
\hline 3.17 & 41.35 \\
\hline 1.64 & 83.49 \\
\hline 1.47 & 97.19 \\
\hline 1.42 & 99.97 \\
\hline 1.1 & 136.07 \\
\hline 1.08 & 138.75 \\
\hline
\end{tabular}

Nota: A. F. Méndez-Sánchez, 2010

Analizamos el comportamiento de un fluido no newtoniano, en este caso, el champú; la determinación del comportamiento viscoso de los fluidos no newtonianos de manera experimental es compleja, puesto que es necesario hacer mediciones en un amplio intervalo de valores de rapidez de deformación (o de esfuerzo de corte). Para este ensayo se tomaron datos experimentales obtenidos del viscosímetro de Couette.

A continuación, se formula la función objetivo:

Donde:

$\hat{\mathrm{y}}_{\mathrm{i}}=\left(\zeta_{0}-\zeta_{\infty}\right)\left[1+(\ddot{e} \times \dot{\gamma})^{a}\right]^{\frac{n-1}{a}}+\zeta_{\infty}$

Por lo tanto, la ecuación 6 quedaría de la siguiente forma: 
$s=\sum_{i=1}^{20}\left(\eta_{i}-\eta_{\infty}-\left(\eta_{0}-\eta_{\infty}\right)\left[1+\left(\lambda \gamma_{i}\right)^{a}\right]^{\frac{n-1}{a}}\right)^{2}$

De acuerdo con el criterio de optimización (minimización) de la función objetivo, se establece lo siguiente: $\frac{d S}{d \eta_{0}}=0, \frac{d S}{d \eta_{\infty}}=0 \quad \frac{d S}{d \lambda}=0, \frac{d S}{d a}=0, \frac{d S}{d n}=0$

\section{Derivando 8 n respecto a se obtiene la siguiente expresión:}

$$
\begin{aligned}
\frac{d S}{d \eta_{0}}=-2 \sum_{i=1}^{20}\left(\eta_{i}-\eta_{\infty}-\left(\eta_{0}-\eta_{\infty}\right) \times\left[1+\left(\lambda \gamma_{i}\right)^{a}\right]^{\frac{n-1}{a}}\right)\left(1+\left(\lambda \gamma_{i}\right)^{a}\right)^{\frac{n-1}{a}}=0 \\
\quad\left(\eta_{1}-\eta_{\infty}-\left(\eta_{0}-\eta_{\infty}\right) \times\left[1+\left(\lambda\left(\dot{\gamma}_{1}\right)\right)^{a}\right]^{\frac{n-1}{a}}\right)\left(1+\left(\lambda\left(\dot{\gamma}_{1}\right)\right)^{a}\right)^{\frac{n-1}{a}}
\end{aligned}
$$

Para $\mathrm{i}=1$ :

Para i=2: $\left(\eta_{2}-\eta_{\infty}-\left(\eta_{0}-\eta_{\infty}\right) \times\left[1+\left(\lambda\left(\dot{\gamma}_{2}\right)\right)^{a}\right]_{n-1}^{\frac{n-1}{a}}\right)\left(1+\left(\lambda\left(\dot{\gamma}_{2}\right)\right)^{a}\right)_{n-1}^{\frac{n-1}{a}}$

Para i=3: $\left(\eta_{3}-\eta_{\infty}-\left(\eta_{0}-\eta_{\infty}\right) \times\left[1+\left(\lambda\left(\dot{\gamma}_{3}\right)\right)^{a}\right]^{\frac{n-1}{a}}\right)\left(1+\left(\lambda\left(\dot{\gamma}_{3}\right)\right)^{a}\right)^{\frac{n-1}{a}}$

Para i=4: $\left(\eta_{4}-\eta_{\infty}-\left(\eta_{0}-\eta_{\infty}\right) \times\left[1+\left(\lambda\left(\dot{\gamma}_{4}\right)\right)^{a}\right]^{\frac{n-1}{a}}\right)\left(1+\left(\lambda\left(\dot{\gamma}_{4}\right)\right)^{a}\right)^{\frac{n-1}{a}}$

Se continúa el procedimiento del mismo modo para los demás puntos; es decir:

Para i=18: $\left(\eta_{18}-\eta_{\infty}-\left(\eta_{0}-\eta_{\infty}\right) \times\left[1+\left(\lambda\left(\dot{\gamma}_{18}\right)\right)^{a}\right]^{\frac{n-1}{a}}\right)\left(1+\left(\lambda\left(\dot{\gamma}_{18}\right)\right)^{a}\right)_{n-1}^{\frac{n-1}{a}}$

Para i=19: $\left(\eta_{19}-\eta_{\infty}-\left(\eta_{0}-\eta_{\infty}\right) \times\left[1+\left(\lambda\left(\dot{\gamma}_{19}\right)\right)^{a}\right]^{\frac{n-1}{a}}\right)\left(1+\left(\lambda\left(\dot{\gamma}_{19}\right)\right)^{a}\right)^{\frac{n-1}{a}}$

Luego, se suman todos los términos tal como indica la ecuación 5.

\section{Derivando $8 \mathbf{n}_{00}$ respecto a se obtiene la siguiente expresión:}

$\frac{d S}{d \eta_{\infty}}=2 \sum_{i=1}^{20}\left(\eta_{i}-\eta_{\infty}-\left(\eta_{0}-\eta_{\infty}\right) \times\left[1+\left(\lambda \gamma_{i}\right)^{a}\right]^{\frac{n-1}{a}}\right)\left(-1+\left[1+\left(\lambda \gamma_{i}\right)^{a}\right]^{\frac{n-1}{a}}\right)=0$

Para $\mathrm{i}=1:\left(\eta_{1}-\eta_{\infty}-\left(\eta_{0}-\eta_{\infty}\right) \times\left[1+\left(\lambda\left(\gamma_{1}\right)\right)^{a}\right]^{\frac{n-1}{a}}\right)\left(-1+\left[1+\left(\lambda\left(\gamma_{1}\right)\right)^{a}\right]^{\frac{n-1}{a}}\right.$

Para i=2: $\left(\eta_{2}-\eta_{\infty}-\left(\eta_{0}-\eta_{\infty}\right) \times\left[1+\left(\lambda\left(\gamma_{2}\right)\right)^{a}\right]^{\frac{n-1}{a}}\right)\left(-1+\left[1+\left(\lambda\left(\gamma_{2}\right)\right)^{a}\right]^{\frac{n-1}{a}}\right)$

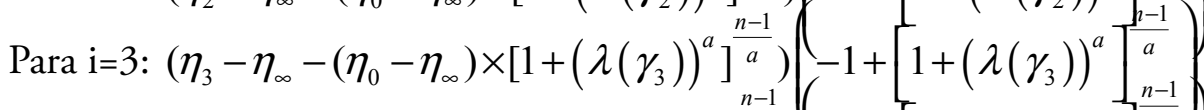

Para i=4: $\left(\eta_{4}-\eta_{\infty}-\left(\eta_{0}-\eta_{\infty}\right) \times\left[1+\left(\lambda\left(\gamma_{4}\right)\right)^{a}\right]^{\frac{n-1}{a}}\right)\left(-1+\left[1+\left(\lambda\left(\gamma_{4}\right)\right)^{a}\right]^{\frac{n-1}{a}}\right)$

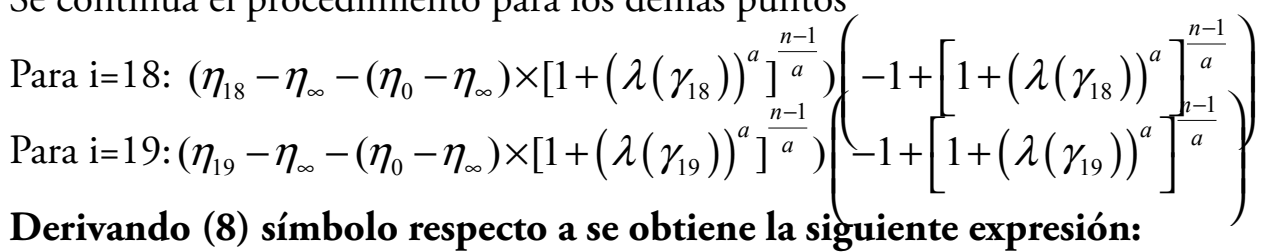

$\frac{d S}{d \lambda}=2 \sum_{i=1}^{20}\left(\eta_{i}-\eta_{\infty}-\left(\eta_{0}-\eta_{\infty}\right) \times\left[1+\left(\lambda \gamma_{i}\right)^{a}\right]^{\frac{n-1}{a}}\right) \times\left(\left(\eta_{\infty}-\eta_{0}\right)\left(\frac{n-1}{a}\right)\left[1+\left(\lambda \gamma_{i}\right)^{a}\right]^{\frac{n-1}{a}-1}(a)\left(\gamma_{i}\right)\left(\lambda \gamma_{i}\right)^{a-1}\right)=0$ 
Para $\mathrm{i}=1$ :

$\left(\eta_{1}-\eta_{\infty}-\left(\eta_{0}-\eta_{\infty}\right) \times\left[1+\left(\lambda\left(\gamma_{1}\right)\right)^{a}\right]^{\frac{n-1}{a}}\right) \times\left(\left(\eta_{\infty}-\eta_{0}\right)\left(\frac{n-1}{a}\right)\left[1+\left(\lambda\left(\gamma_{1}\right)\right)^{a}\right]^{\frac{n-1}{a}-1}(a)\left(\gamma_{1}\right)\left(\lambda\left(\gamma_{1}\right)\right)^{a-1}\right)$
Para i=2:

$\left(\eta_{2}-\eta_{\infty}-\left(\eta_{0}-\eta_{\infty}\right) \times\left[1+\left(\lambda\left(\gamma_{2}\right)\right)^{a}\right]^{\frac{n-1}{a}}\right) \times\left(\left(\eta_{\infty}-\eta_{0}\right)\left(\frac{n-1}{a}\right)\left[1+\left(\lambda\left(\gamma_{2}\right)\right)^{a}\right]^{\frac{n-1}{a}-1}(a)\left(\gamma_{2}\right)\left(\lambda\left(\gamma_{2}\right)\right)^{a-1}\right)$ Para $\mathrm{i}=3$ :

$\left(\eta_{3}-\eta_{\infty}-\left(\eta_{0}-\eta_{\infty}\right) \times\left[1+\left(\lambda\left(\gamma_{3}\right)\right)^{a}\right]^{\frac{n-1}{a}}\right) \times\left(\left(\eta_{\infty}-\eta_{0}\right)\left(\frac{n-1}{a}\right)\left[1+\left(\lambda\left(\gamma_{3}\right)\right)^{a}\right]^{\frac{n-1}{a}-1}(a)\left(\gamma_{3}\right)\left(\lambda\left(\gamma_{3}\right)\right)^{a-1}\right)$

Para $\mathrm{i}=4$ :

$\left.\eta_{4}-\eta_{\infty}-\left(\eta_{0}-\eta_{\infty}\right) \times\left[1+\left(\lambda\left(\gamma_{4}\right)\right)^{a}\right]^{\frac{n-1}{a}}\right) \times\left(\left(\eta_{\infty}-\eta_{0}\right)\left(\frac{n-1}{a}\right)\left[1+\left(\lambda\left(\gamma_{4}\right)\right)^{a}\right]^{\frac{n-1}{a}-1}(a)\left(\gamma_{4}\right)\left(\lambda\left(\gamma_{4}\right)\right)^{a-1}\right)$

Se continúa el procedimiento para los demás datos, tal como indica la ecuación 7.

Para $\mathrm{i}=18$ :

$\left.\eta_{18}-\eta_{\infty}-\left(\eta_{0}-\eta_{\infty}\right) \times\left[1+\left(\lambda\left(\gamma_{18}\right)\right)^{a}\right]^{\frac{n-1}{a}}\right) \times\left(\left(\eta_{\infty}-\eta_{0}\right)\left(\frac{n-1}{a}\right)\left[1+\left(\lambda\left(\gamma_{18}\right)\right)^{a}\right]^{\frac{n-1}{a}-1}(a)\left(\gamma_{18}\right)\left(\lambda\left(\gamma_{18}\right)\right)^{a-1}\right)$

Para i=19:

$\left(\eta_{19}-\eta_{\infty}-\left(\eta_{0}-\eta_{\infty}\right) \times\left[1+\left(\lambda\left(\gamma_{19}\right)\right)^{a}\right]^{\frac{n-1}{a}}\right) \times\left(\left(\eta_{\infty}-\eta_{0}\right)\left(\frac{n-1}{a}\right)\left[1+\left(\lambda\left(\gamma_{19}\right)\right)^{a}\right]^{\frac{n-1}{a}-1}(a)\left(\gamma_{19}\right)\left(\lambda\left(\gamma_{19}\right)\right)^{a-1}\right)$

\section{Derivando 8 alfa respecto a se obtiene la siguiente expresión:}

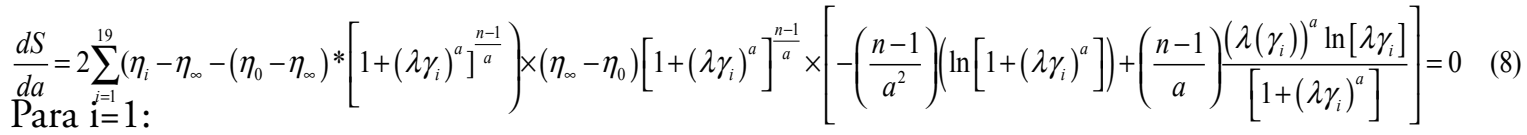
$\left(\eta_{1}-\eta_{\infty}-\left(\eta_{0}-\eta_{\infty}\right)\left[1+\left(\lambda\left(\gamma_{1}\right)\right)^{a}\right]^{\frac{n-1}{a}}\right)\left(\eta_{\infty}-\eta_{0}\right)\left[1+\left(\lambda \times \gamma_{1}\right)^{a}\right]^{\frac{n-1}{a}} \times\left[\left(\frac{n-1}{a}\right) \frac{\left(\lambda \times \gamma_{1}\right)^{a} \ln \left[\lambda \times \gamma_{1}\right]}{\left[1+\left(\lambda \times \gamma_{1}\right)^{a}\right]}-\left(\frac{n-1}{a^{2}}\right)\left(\ln \left[1+\left(\lambda \times \gamma_{1}\right)^{a}\right]\right)\right]$
Para $\mathrm{i}=2:$ $\left(\eta_{2}-\eta_{\infty}-\left(\eta_{0}-\eta_{\infty}\right)\left[1+\left(\lambda\left(\gamma_{2}\right)\right)^{a}\right]^{\frac{n-1}{a}}\right)\left(\eta_{\infty}-\eta_{0}\right)\left[1+\left(\lambda \times \gamma_{2}\right)^{a}\right]^{\frac{n-1}{a}} \times\left[\left(\frac{n-1}{a}\right) \frac{\left(\lambda \times \gamma_{2}\right)^{a} \ln \left[\lambda \times \gamma_{2}\right]}{\left[1+\left(\lambda \times \gamma_{2}\right)^{a}\right]}-\left(\frac{n-1}{a^{2}}\right)\left(\ln \left[1+\left(\lambda \times \gamma_{2}\right)^{a}\right]\right)\right]$
Para i $=3:$ $\left(\eta_{3}-\eta_{\infty}-\left(\eta_{0}-\eta_{\infty}\right)\left[1+\left(\lambda\left(\gamma_{3}\right)\right)^{a}\right]^{a-1}\right)\left(\eta_{\infty}-\eta_{0}\right)\left[1+\left(\lambda \times \gamma_{3}\right)^{a}\right]^{\frac{n-1}{a}} \times\left[\left(\frac{n-1}{a}\right) \frac{\left(\lambda \times \gamma_{3}\right)^{a} \ln \left[\lambda \times \gamma_{3}\right]}{\left[1+\left(\lambda \times \gamma_{3}\right)^{a}\right]}-\left(\frac{n-1}{a^{2}}\right)\left(\ln \left[1+\left(\lambda \times \gamma_{3}\right)^{a}\right]\right)\right]$
Para i $=4:$ $\left(\eta_{4}-\eta_{\infty}-\left(\eta_{0}-\eta_{\infty}\right)\left[1+\left(\lambda\left(\gamma_{4}\right)\right)^{a}\right]^{\frac{n-1}{a}}\right)\left(\eta_{\infty}-\eta_{0}\right)\left[1+\left(\lambda \times \gamma_{4}\right)^{a}\right]^{\frac{n-1}{a}} \times\left[\left(\frac{n-1}{a}\right) \frac{\left(\lambda \times \gamma_{4}\right)^{a} \ln \left[\lambda \times \gamma_{4}\right]}{\left[1+\left(\lambda \times \gamma_{4}\right)^{a}\right]}-\left(\frac{n-1}{a^{2}}\right)\left(\ln \left[1+\left(\lambda \times \gamma_{4}\right)^{a}\right]\right)\right]$

Se continúa el procedimiento para todos los puntos de la ecuación 8.

Para i=18:

$\left(\eta_{18}-\eta_{\infty}-\left(\eta_{0}-\eta_{\infty}\right)\left[1+\left(\lambda\left(\gamma_{18}\right)\right)^{a}\right]^{a-1}\right)\left(\eta_{\infty}-\eta_{0}\right)\left[1+\left(\lambda \times \gamma_{18}\right)^{a}\right]^{\frac{n-1}{a}} \times\left[\left(\frac{n-1}{a}\right) \frac{\left(\lambda \times \gamma_{18}\right)^{a} \ln \left[\lambda \times \gamma_{18}\right]}{\left[1+\left(\lambda \times \gamma_{18}\right)^{a}\right]}-\left(\frac{n-1}{a^{2}}\right)\left(\ln \left[1+\left(\lambda \times \gamma_{18}\right)^{a}\right]\right)\right]$ 
Para $\mathrm{i}=19$ :

$\left(\eta_{19}-\eta_{\infty}-\left(\eta_{0}-\eta_{\infty}\right)\left[1+\left(\lambda\left(\gamma_{19}\right)\right)^{a}\right]^{a-1}\right)\left(\eta_{\infty}-\eta_{0}\right)\left[1+\left(\lambda \times \gamma_{19}\right)^{a}\right]^{\frac{n-1}{a}} \times\left[\left(\frac{n-1}{a}\right) \frac{\left(\lambda \times \gamma_{19}\right)^{a} \ln \left[\lambda \times \gamma_{19}\right]}{\left[1+\left(\lambda \times \gamma_{19}\right)^{a}\right]}-\left(\frac{n-1}{a^{2}}\right)\left(\ln \left[1+\left(\lambda \times \gamma_{19}\right)^{a}\right]\right)\right]$

\section{Derivando (8) n respecto a se obtiene la siguiente expresión:}

$\frac{d S}{d n}=2 \sum_{i=1}^{19}\left(\eta_{i}-\eta_{\infty}-\left(\eta_{0}-\eta_{\infty}\right)\left[1+\left(\lambda \gamma_{i}\right)^{a}\right]^{\frac{n-1}{a}}\right)\left(\left(\eta_{\infty}-\eta_{0}\right)\left(\frac{1}{a}\right)\left[1+\left(\lambda \gamma_{i}\right)^{a}\right]^{\frac{n-1}{a}}\left(\ln \left[1+\left(\lambda \gamma_{i}\right)^{a}\right]\right)\right)=0$

Para i $=1:\left(\eta_{1}-\eta_{\infty}-\left(\eta_{0}-\eta_{\infty}\right)\left[1+\left(\lambda\left(\gamma_{1}\right)\right)^{a}\right]^{\frac{n-1}{a}}\right)\left(\left(\eta_{\infty}-\eta_{0}\right)\left(\frac{1}{a}\right)\left[1+\left(\lambda\left(\gamma_{1}\right)\right)^{a}\right]^{\frac{n-1}{a}}\left(\ln \left[1+\left(\lambda\left(\gamma_{1}\right)\right)^{a}\right]\right)\right)$

Para i $=2:\left(\eta_{2}-\eta_{\infty}-\left(\eta_{0}-\eta_{\infty}\right)\left[1+\left(\lambda\left(\gamma_{2}\right)\right)^{a}\right]^{\frac{n-1}{a}}\right)\left(\left(\eta_{\infty}-\eta_{0}\right)\left(\frac{1}{a}\right)\left[1+\left(\lambda\left(\gamma_{2}\right)\right)^{a}\right]^{\frac{n-1}{a}}\left(\ln \left[1+\left(\lambda\left(\gamma_{2}\right)\right)^{a}\right]\right)\right)$

Para i=3: $\left(\eta_{3}-\eta_{\infty}-\left(\eta_{0}-\eta_{\infty}\right)\left[1+\left(\lambda\left(\gamma_{3}\right)\right)^{a}\right]^{\frac{n-1}{a}}\right)\left(\left(\eta_{\infty}-\eta_{0}\right)\left(\frac{1}{a}\right)\left[1+\left(\lambda\left(\gamma_{3}\right)\right)^{a}\right]^{\frac{n-1}{a}}\left(\ln \left[1+\left(\lambda\left(\gamma_{3}\right)\right)^{a}\right]\right)\right)$

Para i $=4:\left(\eta_{4}-\eta_{\infty}-\left(\eta_{0}-\eta_{\infty}\right)\left[1+\left(\lambda\left(\gamma_{4}\right)\right)^{a}\right]^{\frac{n-1}{a}}\right)\left(\left(\eta_{\infty}-\eta_{0}\right)\left(\frac{1}{a}\right)\left[1+\left(\lambda\left(\gamma_{4}\right)\right)^{a}\right]^{\frac{n-1}{a}}\left(\ln \left[1+\left(\lambda\left(\gamma_{4}\right)\right)^{a}\right]\right)\right)$

Se continúa el procedimiento para todos los puntos dados, de acuerdo a la ecuación 9

Para i=18: $\left(\eta_{18}-\eta_{\infty}-\left(\eta_{0}-\eta_{\infty}\right)\left[1+\left(\lambda\left(\gamma_{18}\right)\right)^{a}\right]^{\frac{n-1}{a}}\right)\left(\left(\eta_{\infty}-\eta_{0}\right)\left(\frac{1}{a}\right)\left[1+\left(\lambda\left(\gamma_{18}\right)\right)^{a}\right]^{\frac{n-1}{a}}\left(\ln \left[1+\left(\lambda\left(\gamma_{18}\right)\right)^{a}\right]\right)\right)$

Para i=19: $\left(\eta_{19}-\eta_{\infty}-\left(\eta_{0}-\eta_{\infty}\right)\left[1+\left(\lambda\left(\gamma_{19}\right)\right)^{a}\right]^{\frac{n-1}{a}}\right)\left(\left(\eta_{\infty}-\eta_{0}\right)\left(\frac{1}{a}\right)\left[1+\left(\lambda\left(\gamma_{19}\right)\right)^{a}\right]^{\frac{n-1}{a}}\left(\ln \left[1+\left(\lambda\left(\gamma_{19}\right)\right)^{a}\right]\right)\right)$

Finalmente, se obtiene un sistema de cinco ecuaciones algebraicas no lineales cada una de las cuales contiene 20 términos.

Al hacer la regresión debemos verificar la validez del modelo mediante el cálculo del coeficiente de regresión múltiple, dado por:

$$
R^{2}=1-\frac{\sum_{1}^{19}\left(y_{i}-\hat{y}_{i}\right)^{2}}{\sum_{1}^{19}\left(y_{i}-\bar{y}\right)^{2}}
$$




\section{Resultados}

En primer lugar, se hacen las gráficas de la velocidad de corte y la viscosidad, además del esfuerzo de corte y la velocidad de corte experimentales, los cuales muestran el comportamiento típico de un fluido no newtoniano.

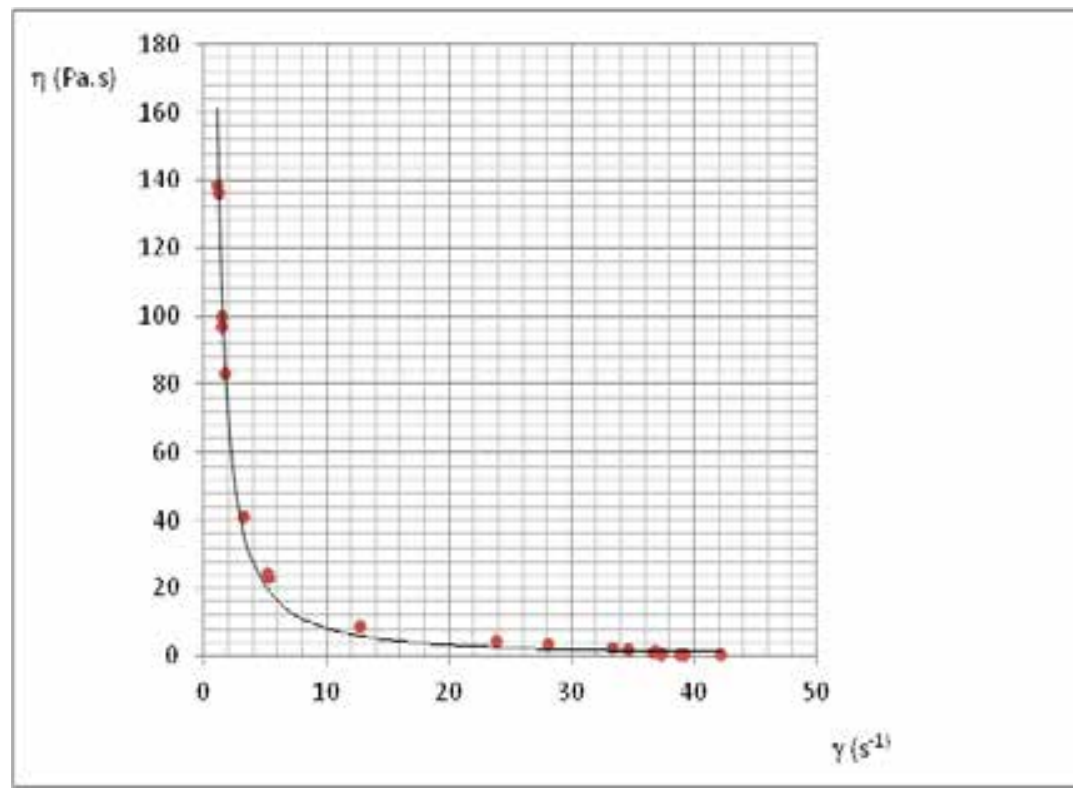

Figura 3: Dependencia entre la velocidad de corte y la viscosidad Fuente: Elaborada a partir de los datos experimentales dados en la Tabla 1

En la Figura 3, se observa que a valores de velocidad de corte altos, el champú se comporta como un fluido newtoniano; mientras que para valores intermedios, dicho comportamiento obedece al de un fluido no newtoniano.

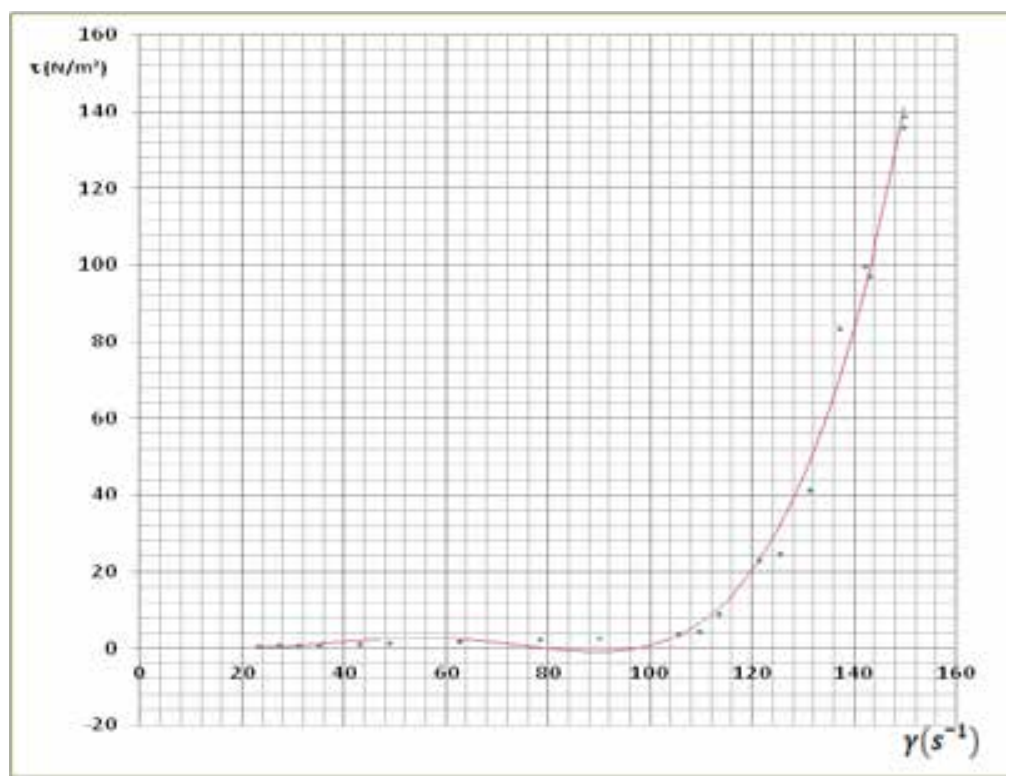

Figura 4. Dependencia entre la velocidad de corte y el esfuerzo de corte

Fuente: Elaborada a partir de los datos experimentales dados en la Tabla 1. El valor de $\tau=\eta_{a} \times \dot{\gamma} ; \quad$ ( $\eta_{a}$ y $\left.\dot{\gamma}\right)$, está dado en la Tabla antes indicada. 
Con las cinco ecuaciones algebraicas no lineales dadas, se elaboró y ejecutó el programa denominado "Carreu-Yasuda regresión 1.pol", que se encuentra en el anexo respectivo, y se obtienen los siguientes valores de los parámetros del modelo de Carreu-Yasuda.

Tabla 2

Resultados de la ejecución del programa "Carreu-Yasuda regresión 1.pol"

\begin{tabular}{|c|c|}
\hline Variable & Valor \\
\hline $\mathbf{A}=\eta_{0}$ & 38.105010 \\
\hline $\mathbf{B}=\eta_{\infty}$ & 0.4760314 \\
\hline $\mathbf{C}=\lambda$ & 0.3127583 \\
\hline $\mathbf{D}=a$ & 3.2708100 \\
\hline $\mathbf{E}=n$ & -0.0564591 \\
\hline
\end{tabular}

Cabe mencionar, que el cuarto dato de la Tabla 1 no ha sido tomado en cuenta, por la inspección de las Figuras 1 y 2, lo que evidencia que este dato no se encuentra dentro de los valores esperados.

El programa de cálculo respectivo, se adjunta en otro documento adicional.

Los valores reportados en la bibliografía (Méndez-Sánchez et al., 2010), son los siguientes:

Tabla 3

Valores reportados

\begin{tabular}{|c|c|}
\hline$\eta_{0}$ & $38.97207 \pm 0.61803$ \\
\hline$\eta_{\infty}$ & 0 \\
\hline$\lambda$ & $0.32487 \pm 0.04182$ \\
\hline$a$ & $2.95998 \pm 0.70741$ \\
\hline$n$ & $0.00978 \pm 0.09873$ \\
\hline
\end{tabular}

Nota: Extraído de Méndez-Sánches et al., 2010.
Dichos autores indican que los paráme- tros del modelo han sido calculados mediante el software Origin que utiliza el algoritmo de Levenberg-Maquard; sin embargo, no explican por qué no se calculó el valor de y más bien asignan el valor de cero, tal como indica la tabla anterior.

El software Polymath tiene un utilitario de tratamiento estadístico de datos; mediante este software, se realizó la regresión respectiva y se obtienen los valores de los parámetros que se muestran en la Tabla 4. El archivo se denomina Carreu-Yasuda regresión 2.pol”

Se ingresa la función de regresión $y=(A-B) \times\left(1+(C \times x)^{D}\right)^{(E-1) / D}+B$

Tabla 4

Reporte de los datos usando el utilitario estadistico del software Polymath

\begin{tabular}{|c|c|c|}
\hline Variable & Valor & $\begin{array}{c}95 \% \text { de } \\
\text { confianza }\end{array}$ \\
\hline $\mathbf{A}$ & 38.10502 & 0.0132452 \\
\hline B & 0.476010 & 0.0137652 \\
\hline C & 0.312759 & 0.0003522 \\
\hline D & 3.270811 & 0.0098815 \\
\hline $\mathbf{E}$ & -0.05645 & 0.0015279 \\
\hline
\end{tabular}

Varianza $=0.3243332$

Comparando las Tablas 2 y 4 , se observa que tanto el cálculo de regresión usado, resolviendo un sistema de ecuaciones algebraicas no lineales, así como el obtenido mediante el utilitario estadístico del Polymath, reportan los mismos valores. 
Tabla 5

Valores de la variable dependiente $(\eta)$ observados (experimentales) y estimados (usando el modelo de Carreu-Yasuda, y los parámetros obtenidos por regresión) y los valores residuales obtenidos por diferencia entre los observados y los estimados

\begin{tabular}{|c|c|c|c|}
\hline $\mathbf{X}=\dot{\gamma}$ & $\mathbf{Y}_{\text {obs. }}$ & $\mathbf{Y}_{\text {calc. }}$ & Residual \\
\hline 0.624 & 37.211 & 38.04717 & $\begin{array}{c}- \\
0.8361654\end{array}$ \\
\hline 0.700 & 38.771 & 38.02088 & 0.7501158 \\
\hline 0.792 & 39.229 & 37.9793 & 1.2497000 \\
\hline 1.170 & 36.623 & 37.66231 & $\begin{array}{c}- \\
1.039312 \\
\end{array}$ \\
\hline 1.310 & 37.198 & 37.47106 & $\begin{array}{c}- \\
0.2730644 \\
\end{array}$ \\
\hline 1.70 & 36.747 & 36.68300 & 0.064000 \\
\hline 2.26 & 34.592 & 34.86514 & $\begin{array}{c}- \\
0.2731417 \\
\end{array}$ \\
\hline 2.70 & 33.310 & 32.96844 & 0.3415563 \\
\hline 3.77 & 28.020 & 27.73218 & 0.2878162 \\
\hline 4.61 & 23.770 & 23.95097 & $\begin{array}{c}- \\
0.1809701\end{array}$ \\
\hline 8.92 & 12.720 & 13.06479 & $\begin{array}{c}- \\
0.3447858 \\
\end{array}$ \\
\hline 23.20 & 5.230 & 5.110697 & 0.1193031 \\
\hline 24.70 & 5.070 & 4.814265 & 0.2557347 \\
\hline 41.35 & 3.170 & 2.993953 & 0.1760473 \\
\hline 83.49 & 1.640 & 1.674650 & $\begin{array}{c}- \\
0.0346501\end{array}$ \\
\hline 97.19 & 1.470 & 1.496897 & $\begin{array}{c}- \\
0.0268972 \\
\end{array}$ \\
\hline 99.97 & 1.420 & 1.466930 & $\begin{array}{c}- \\
0.0469295 \\
\end{array}$ \\
\hline 136.07 & 1.100 & 1.191475 & $\begin{array}{c}- \\
0.0914746 \\
\end{array}$ \\
\hline 138.75 & 1.080 & 1.176883 & $\begin{array}{c}- \\
0.0968831\end{array}$ \\
\hline
\end{tabular}

Viscosidad observada y estimada del modelo de Carreu-Yasuda vs velocidad de cizallamiento

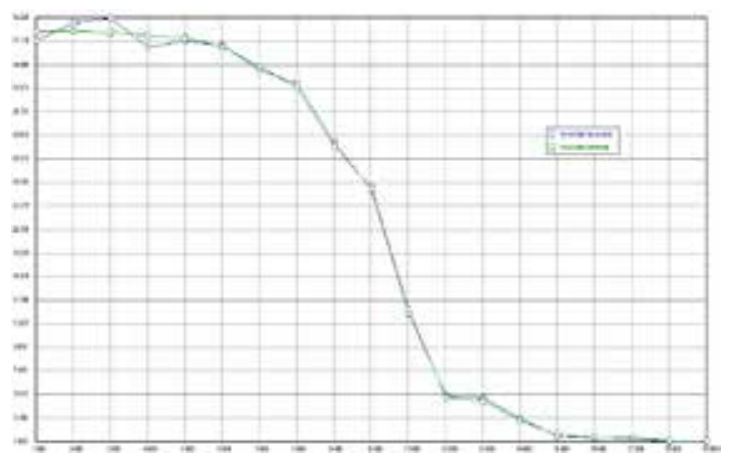

Figura 5: Viscosidad observada y viscosidad estimada, obtenida a base de los datos de la Tabla 5 .

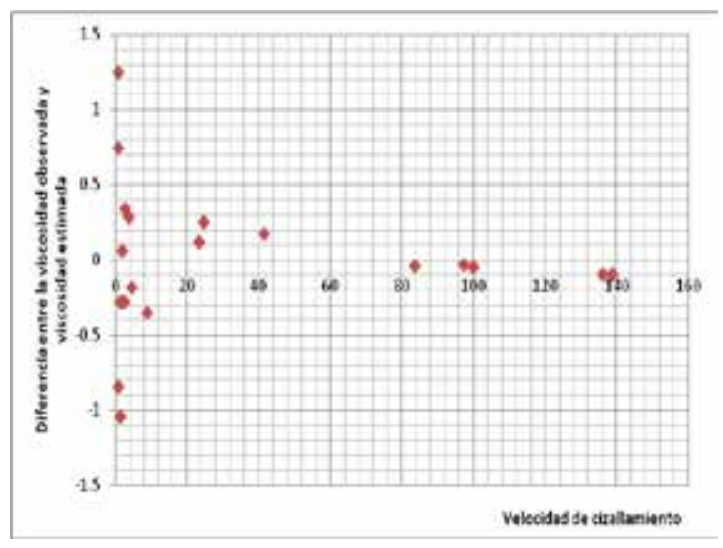

Figura 6. Valores residuales entre la viscosidad observada y viscosidad estimada frente a la velocidad de cizallamiento.

\section{Discusión}

Se han analizado las diferencias existentes entre fluidos newtonianos y no newtonianos, y se mostraron las respectivas curvas típicas de diversos modelos reológicos que caracterizan a un determinado grupo de fluidos, que presentan dos o más parámetros, que deben ser obtenidos por métodos de regresión no lineal.

De los reogramas observados en las Figuras 1 y 2 , se puede deducir que el champú es un fluido no newtoniano adelgazante; $y$ 
presenta una comportamiento atípico, pues a velocidades de corte muy bajo tiene un comportamiento newtoniano. Este comportamiento newtoniano, también ocurre a velocidades de corte alto, mientras que a velocidades de corte moderado, presenta el comportamiento típico de un fluido no newtoniano, vale decir que la dependencia entre la velocidad de corte y el esfuerzo de corte es no lineal.

En el cálculo de parámetros del modelo de Carreu-Yasuda, que en este caso son cinco, se ha utilizado el método de regresión no lineal, cuyos resultados fueron comparados con los obtenidos mediante un software como es el Polymath y con los valores reportados en la literatura por Méndez-Sánchez et al., 2010 y cuyas diferencias son mínimas.

Se presenta el método de regresión desarrollado y el programa de cálculo utilizado para resolver el sistema de cinco ecuaciones algebraicas resultantes partiendo de los valores iniciales dados, a base de las curvas de los datos experimentales.

\section{Referencias}

Carrasco, L. (2011). Métodos numéricos aplicados a la ingeniería ( $3^{\circ}$ ed.). Lima, Perú: Macro.

Carrillo, A. (2011). Arrastre incipiente de partículas en flujo de fluidos o newtonianos (Tesis de pregrado, Universidad de Chile, Santiago de Chile).
Méndez-Sánchez, A., Pérez, L. y Paniagua, A. (enero, 2010). Determinación de la viscosidad de fluidos newtonianos $y$ no newtonianos (una revisión del viscosimetro de Couette). Latin-American Journal of Physics Education, 4(1), 237-245. 


\title{
ANEXO: PROGRAMA DE CÁLCULO DESARROLLADO EN POLYMATH
}

\section{Este programa permite calcular los parámetros del modelo de Carreu-Yasuda}

\section{(Carreu-Yasuda reresión1.pol)}

\author{
\# Estas funciones definen las derivadas parciales de la función objetivo, respecto de cada uno de los \\ parámetros del modelo, que en este caso son cinco. \\ $\mathrm{f}(\mathrm{A})=2 *(\mathrm{~A} 1+\mathrm{A} 2+\mathrm{A} 3+\mathrm{A} 4+\mathrm{A} 5+\mathrm{A} 6+\mathrm{A} 7+\mathrm{A} 8+\mathrm{A} 9+\mathrm{A} 10+\mathrm{A} 11+\mathrm{A} 12+\mathrm{A} 13+\mathrm{A} 14+\mathrm{A} 15+\mathrm{A} 16+\mathrm{A} 17$ \\ $+\mathrm{A} 18+\mathrm{A} 19)$ \\ $\mathrm{f}(\mathrm{B})=2 *(\mathrm{~B} 1+\mathrm{B} 2+\mathrm{B} 3+\mathrm{B} 4+\mathrm{B} 5+\mathrm{B} 6+\mathrm{B} 7+\mathrm{B} 8+\mathrm{B} 9+\mathrm{B} 10+\mathrm{B} 11+\mathrm{B} 12+\mathrm{B} 13+\mathrm{B} 14+\mathrm{B} 15+\mathrm{B} 16+\mathrm{B} 17$ \\ $+\mathrm{B} 18+\mathrm{B} 19)$ \\ $\mathrm{f}(\mathrm{C})=2 *(\mathrm{C} 1+\mathrm{C} 2+\mathrm{C} 3+\mathrm{C} 4+\mathrm{C} 5+\mathrm{C} 6+\mathrm{C} 7+\mathrm{C} 8+\mathrm{C} 9+\mathrm{C} 10+\mathrm{C} 11+\mathrm{C} 12+\mathrm{C} 13+\mathrm{C} 14+\mathrm{C} 15+\mathrm{C} 16+$ \\ $\mathrm{C} 17+\mathrm{C} 18+\mathrm{C} 19)$ \\ $\mathrm{f}(\mathrm{D})=2 *(\mathrm{D} 1+\mathrm{D} 2+\mathrm{D} 3+\mathrm{D} 4+\mathrm{D} 5+\mathrm{D} 6+\mathrm{D} 7+\mathrm{D} 8+\mathrm{D} 9+\mathrm{D} 10+\mathrm{D} 11+\mathrm{D} 12+\mathrm{D} 13+\mathrm{D} 14+\mathrm{D} 15+\mathrm{D} 16$ \\ $+\mathrm{D} 17+\mathrm{D} 18+\mathrm{D} 19)$ \\ $\mathrm{f}(\mathrm{E})=2 *(\mathrm{E} 1+\mathrm{E} 2+\mathrm{E} 3+\mathrm{E} 4+\mathrm{E} 5+\mathrm{E} 6+\mathrm{E} 7+\mathrm{E} 8+\mathrm{E} 9+\mathrm{E} 10+\mathrm{E} 11+\mathrm{E} 12+\mathrm{E} 13+\mathrm{E} 14+\mathrm{E} 15+\mathrm{E} 16+\mathrm{E} 17+$ \\ $\mathrm{E} 18+\mathrm{E} 19)$ \\ \# Valores iniciales de prueba \\ $\mathrm{A}(0)=33.07$ \\ $\mathrm{B}(0)=0.2$ \\ $\mathrm{C}(0)=0.3125$ \\ $\mathrm{D}(0)=2$ \\ $\mathrm{E}(0)=0.1062$ \\ \# Expansión de cada uno de los términos de la derivada de la función respecto al parámetro A \\ $\mathrm{A} 1=(\mathrm{Y} 1-\mathrm{B}-(\mathrm{A}-\mathrm{B}) *((1+((\mathrm{C} * \mathrm{X} 1) \wedge \mathrm{D})) \wedge((\mathrm{E}-1) / \mathrm{D})))^{*}((1+((\mathrm{C} * \mathrm{X} 1) \wedge \mathrm{D})) \wedge((\mathrm{E}-1) / \mathrm{D}))$ \\ $\mathrm{A} 2=(\mathrm{Y} 2-\mathrm{B}-(\mathrm{A}-\mathrm{B}) *((1+((\mathrm{C} * \mathrm{X} 2) \wedge \mathrm{D})) \wedge((\mathrm{E}-1) / \mathrm{D}))) *((1+((\mathrm{C} * \mathrm{X} 2) \wedge \mathrm{D})) \wedge((\mathrm{E}-1) / \mathrm{D}))$ \\ $\mathrm{A} 3=(\mathrm{Y} 3-\mathrm{B}-(\mathrm{A}-\mathrm{B}) *((1+((\mathrm{C} * \mathrm{X} 3) \wedge \mathrm{D})) \wedge((\mathrm{E}-1) / \mathrm{D})))^{*}\left(\left(1+\left((\mathrm{C} * \mathrm{X} 3)^{\wedge} \mathrm{D}\right)\right) \wedge((\mathrm{E}-1) / \mathrm{D})\right)$ \\ $\mathrm{A} 4=\left(\mathrm{Y} 4-\mathrm{B}-(\mathrm{A}-\mathrm{B})^{*}((1+((\mathrm{C} * \mathrm{X} 4) \wedge \mathrm{D})) \wedge((\mathrm{E}-1) / \mathrm{D}))\right) *((1+((\mathrm{C} * \mathrm{X} 4) \wedge \mathrm{D})) \wedge((\mathrm{E}-1) / \mathrm{D}))$ \\ $\mathrm{A} 5=(\mathrm{Y} 5-\mathrm{B}-(\mathrm{A}-\mathrm{B}) *((1+((\mathrm{C} * \mathrm{X} 5) \wedge \mathrm{D})) \wedge((\mathrm{E}-1) / \mathrm{D})))^{*}\left(\left(1+\left((\mathrm{C} * \mathrm{X} 5)^{\wedge} \mathrm{D}\right)\right) \wedge((\mathrm{E}-1) / \mathrm{D})\right)$ \\ $\mathrm{A} 6=(\mathrm{Y} 6-\mathrm{B}-(\mathrm{A}-\mathrm{B}) *((1+((\mathrm{C} * \mathrm{X} 6) \wedge \mathrm{D})) \wedge((\mathrm{E}-1) / \mathrm{D}))) *((1+((\mathrm{C} * \mathrm{X} 6) \wedge \mathrm{D})) \wedge((\mathrm{E}-1) / \mathrm{D}))$ \\ $\mathrm{A} 7=(\mathrm{Y} 7-\mathrm{B}-(\mathrm{A}-\mathrm{B}) *((1+((\mathrm{C} * \mathrm{X} 7) \wedge \mathrm{D})) \wedge((\mathrm{E}-1) / \mathrm{D}))) *((1+((\mathrm{C} * \mathrm{X} 7) \wedge \mathrm{D})) \wedge((\mathrm{E}-1) / \mathrm{D}))$ \\ $\mathrm{A} 8=\left(\mathrm{Y} 8-\mathrm{B}-(\mathrm{A}-\mathrm{B}) *\left(\left(1+\left((\mathrm{C} * \mathrm{X} 8)^{\wedge} \mathrm{D}\right)\right) \wedge((\mathrm{E}-1) / \mathrm{D})\right)\right)^{*}\left(\left(1+\left((\mathrm{C} * \mathrm{X} 8)^{\wedge} \mathrm{D}\right)\right) \wedge((\mathrm{E}-1) / \mathrm{D})\right)$ \\ $\mathrm{A} 9=(\mathrm{Y} 9-\mathrm{B}-(\mathrm{A}-\mathrm{B}) *((1+((\mathrm{C} * \mathrm{X} 9) \wedge \mathrm{D})) \wedge((\mathrm{E}-1) / \mathrm{D})))^{*}((1+((\mathrm{C} * \mathrm{X} 9) \wedge \mathrm{D})) \wedge((\mathrm{E}-1) / \mathrm{D}))$ \\ $\mathrm{A} 10=(\mathrm{Y} 10-\mathrm{B}-(\mathrm{A}-\mathrm{B}) *((1+((\mathrm{C} * \mathrm{X} 10) \wedge \mathrm{D})) \wedge((\mathrm{E}-1) / \mathrm{D})))^{*}((1+((\mathrm{C} * \mathrm{X} 10) \wedge \mathrm{D})) \wedge((\mathrm{E}-1) / \mathrm{D}))$ \\ $\mathrm{A} 11=(\mathrm{Y} 11-\mathrm{B}-(\mathrm{A}-\mathrm{B}) *((1+((\mathrm{C} * \mathrm{X} 11) \wedge \mathrm{D})) \wedge((\mathrm{E}-1) / \mathrm{D}))) *((1+((\mathrm{C} * \mathrm{X} 11) \wedge \mathrm{D})) \wedge((\mathrm{E}-1) / \mathrm{D}))$ \\ $\mathrm{A} 12=(\mathrm{Y} 12-\mathrm{B}-(\mathrm{A}-\mathrm{B}) *((1+((\mathrm{C} * \mathrm{X} 12) \wedge \mathrm{D})) \wedge((\mathrm{E}-1) / \mathrm{D}))) *((1+((\mathrm{C} * \mathrm{X} 12) \wedge \mathrm{D})) \wedge((\mathrm{E}-1) / \mathrm{D}))$
}




$$
\begin{aligned}
& \mathrm{A} 13=\left(\mathrm{Y} 13-\mathrm{B}-(\mathrm{A}-\mathrm{B})^{*}\left(\left(1+\left(\left(\mathrm{C}^{*} \mathrm{X} 13\right) \wedge \mathrm{D}\right)\right)^{\wedge}((\mathrm{E}-1) / \mathrm{D})\right)\right)^{*}((1+((\mathrm{C} * \mathrm{X} 13) \wedge \mathrm{D})) \wedge((\mathrm{E}-1) / \mathrm{D})) \\
& \mathrm{A} 14=\left(\mathrm{Y} 14-\mathrm{B}-(\mathrm{A}-\mathrm{B})^{*}\left(\left(1+\left(\left(\mathrm{C}^{*} \mathrm{X} 14\right) \wedge \mathrm{D}\right)\right)^{\wedge}((\mathrm{E}-1) / \mathrm{D})\right)\right)^{*}((1+((\mathrm{C} * \mathrm{X} 14) \wedge \mathrm{D})) \wedge((\mathrm{E}-1) / \mathrm{D})) \\
& \mathrm{A} 15=\left(\mathrm{Y} 15-\mathrm{B}-(\mathrm{A}-\mathrm{B})^{*}\left(\left(1+\left(\left(\mathrm{C}^{*} \mathrm{X} 15\right) \wedge \mathrm{D}\right)\right)^{\wedge}((\mathrm{E}-1) / \mathrm{D})\right)\right)^{*}((1+((\mathrm{C} * \mathrm{X} 15) \wedge \mathrm{D})) \wedge((\mathrm{E}-1) / \mathrm{D})) \\
& \mathrm{A} 16=\left(\mathrm{Y} 16-\mathrm{B}-(\mathrm{A}-\mathrm{B})^{*}\left(\left(1+\left(\left(\mathrm{C}^{*} \mathrm{X} 16\right) \wedge \mathrm{D}\right)\right)^{\wedge}((\mathrm{E}-1) / \mathrm{D})\right)\right)^{*}((1+((\mathrm{C} * \mathrm{X} 16) \wedge \mathrm{D})) \wedge((\mathrm{E}-1) / \mathrm{D})) \\
& \mathrm{A} 17=\left(\mathrm{Y} 17-\mathrm{B}-(\mathrm{A}-\mathrm{B})^{*}\left((1+((\mathrm{C} * \mathrm{X} 17) \wedge \mathrm{D}))^{\wedge}((\mathrm{E}-1) / \mathrm{D})\right)\right)^{*}((1+((\mathrm{C} * \mathrm{X} 17) \wedge \mathrm{D})) \wedge((\mathrm{E}-1) / \mathrm{D})) \\
& \mathrm{A} 18=\left(\mathrm{Y} 18-\mathrm{B}-(\mathrm{A}-\mathrm{B}) *\left((1+((\mathrm{C} * \mathrm{X} 18) \wedge \mathrm{D}))^{\wedge}((\mathrm{E}-1) / \mathrm{D})\right)\right)^{*}((1+((\mathrm{C} * \mathrm{X} 18) \wedge \mathrm{D})) \wedge((\mathrm{E}-1) / \mathrm{D})) \\
& \mathrm{A} 19=\left(\mathrm{Y} 19-\mathrm{B}-(\mathrm{A}-\mathrm{B})^{*}\left((1+((\mathrm{C} * \mathrm{X} 19) \wedge \mathrm{D}))^{\wedge}((\mathrm{E}-1) / \mathrm{D})\right)\right)^{*}((1+((\mathrm{C} * \mathrm{X} 19) \wedge \mathrm{D})) \wedge((\mathrm{E}-1) / \mathrm{D}))
\end{aligned}
$$$$
\text { \# Expansión de cada uno de los términos de la derivada de la función respecto al parámetro B }
$$$$
\mathrm{B} 1=\left(\mathrm{Y} 1-\mathrm{B}-(\mathrm{A}-\mathrm{B})^{*}\left(\left(1+\left((\mathrm{C} * \mathrm{X} 1)^{\wedge} \mathrm{D}\right)\right)^{\wedge}((\mathrm{E}-1) / \mathrm{D})\right)\right)^{*}\left(-1+\left(\left(1+\left((\mathrm{C} * \mathrm{X} 1)^{\wedge} \mathrm{D}\right)\right)^{\wedge}((\mathrm{E}-1) / \mathrm{D})\right)\right)
$$$$
\mathrm{B} 2=\left(\mathrm{Y} 2-\mathrm{B}-(\mathrm{A}-\mathrm{B}) *\left((1+((\mathrm{C} * \mathrm{X} 2) \wedge \mathrm{D}))^{\wedge}((\mathrm{E}-1) / \mathrm{D})\right)\right)^{*}(-1+((1+((\mathrm{C} * \mathrm{X} 2) \wedge \mathrm{D})) \wedge((\mathrm{E}-1) / \mathrm{D})))
$$$$
\mathrm{B} 3=\left(\mathrm{Y} 3-\mathrm{B}-(\mathrm{A}-\mathrm{B})^{*}\left(\left(1+\left(\left(\mathrm{C}^{*} \mathrm{X} 3\right)^{\wedge} \mathrm{D}\right)\right)^{\wedge}((\mathrm{E}-1) / \mathrm{D})\right)\right)^{*}\left(-1+\left((1+((\mathrm{C} * \mathrm{X} 3) \wedge \mathrm{D}))^{\wedge}((\mathrm{E}-1) / \mathrm{D})\right)\right)
$$$$
\mathrm{B} 4=\left(\mathrm{Y} 4-\mathrm{B}-(\mathrm{A}-\mathrm{B}) *\left((1+((\mathrm{C} * \mathrm{X} 4) \wedge \mathrm{D}))^{\wedge}((\mathrm{E}-1) / \mathrm{D})\right)\right)^{*}\left(-1+\left((1+((\mathrm{C} * \mathrm{X} 4) \wedge \mathrm{D}))^{\wedge}((\mathrm{E}-1) / \mathrm{D})\right)\right)
$$$$
\mathrm{B} 5=\left(\mathrm{Y} 5-\mathrm{B}-(\mathrm{A}-\mathrm{B}) *\left((1+((\mathrm{C} * \mathrm{X} 5) \wedge \mathrm{D}))^{\wedge}((\mathrm{E}-1) / \mathrm{D})\right)\right)^{*}(-1+((1+((\mathrm{C} * \mathrm{X} 5) \wedge \mathrm{D})) \wedge((\mathrm{E}-1) / \mathrm{D})))
$$$$
\mathrm{B} 6=\left(\mathrm{Y} 6-\mathrm{B}-(\mathrm{A}-\mathrm{B}) *\left((1+((\mathrm{C} * \mathrm{X} 6) \wedge \mathrm{D}))^{\wedge}((\mathrm{E}-1) / \mathrm{D})\right)\right)^{*}(-1+((1+((\mathrm{C} * \mathrm{X} 6) \wedge \mathrm{D})) \wedge((\mathrm{E}-1) / \mathrm{D})))
$$$$
\mathrm{B} 7=\left(\mathrm{Y} 7-\mathrm{B}-(\mathrm{A}-\mathrm{B}) *\left((1+((\mathrm{C} * \mathrm{X} 7) \wedge \mathrm{D}))^{\wedge}((\mathrm{E}-1) / \mathrm{D})\right)\right)^{*}(-1+((1+((\mathrm{C} * \mathrm{X} 7) \wedge \mathrm{D})) \wedge((\mathrm{E}-1) / \mathrm{D})))
$$$$
\mathrm{B} 8=\left(\mathrm{Y} 8-\mathrm{B}-(\mathrm{A}-\mathrm{B}) *\left(\left(1+\left((\mathrm{C} * \mathrm{X} 8)^{\wedge} \mathrm{D}\right)\right)^{\wedge}((\mathrm{E}-1) / \mathrm{D})\right)\right)^{*}(-1+((1+((\mathrm{C} * \mathrm{X} 8) \wedge \mathrm{D})) \wedge((\mathrm{E}-1) / \mathrm{D})))
$$$$
\mathrm{B} 9=\left(\mathrm{Y} 9-\mathrm{B}-(\mathrm{A}-\mathrm{B}) *\left((1+((\mathrm{C} * \mathrm{X} 9) \wedge \mathrm{D}))^{\wedge}((\mathrm{E}-1) / \mathrm{D})\right)\right)^{*}\left(-1+\left((1+((\mathrm{C} * \mathrm{X} 9) \wedge \mathrm{D}))^{\wedge}((\mathrm{E}-1) / \mathrm{D})\right)\right)
$$$$
\mathrm{B} 10=\left(\mathrm{Y} 10-\mathrm{B}-(\mathrm{A}-\mathrm{B})^{*}\left(\left(1+\left(\left(\mathrm{C}^{*} \mathrm{X} 10\right) \wedge \mathrm{D}\right)\right)^{\wedge}((\mathrm{E}-1) / \mathrm{D})\right)\right)^{*}\left(-1+\left(\left(1+\left((\mathrm{C} * \mathrm{X} 10)^{\wedge} \mathrm{D}\right)\right)^{\wedge}((\mathrm{E}-1) / \mathrm{D})\right)\right)
$$$$
\mathrm{B} 11=\left(\mathrm{Y} 11-\mathrm{B}-(\mathrm{A}-\mathrm{B}) *\left(\left(1+\left(\left(\mathrm{C}^{*} \mathrm{X} 11\right) \wedge \mathrm{D}\right)\right)^{\wedge}((\mathrm{E}-1) / \mathrm{D})\right)\right)^{*}\left(-1+\left((1+((\mathrm{C} * \mathrm{X} 11) \wedge \mathrm{D}))^{\wedge}((\mathrm{E}-1) / \mathrm{D})\right)\right)
$$$$
\mathrm{B} 12=\left(\mathrm{Y} 12-\mathrm{B}-(\mathrm{A}-\mathrm{B}) *\left((1+((\mathrm{C} * \mathrm{X} 12) \wedge \mathrm{D}))^{\wedge}((\mathrm{E}-1) / \mathrm{D})\right)\right)^{*}\left(-1+\left(\left(1+\left(\left(\mathrm{C}^{*} \mathrm{X} 12\right) \wedge \mathrm{D}\right)\right)^{\wedge}((\mathrm{E}-1) / \mathrm{D})\right)\right)
$$$$
\mathrm{B} 13=\left(\mathrm{Y} 13-\mathrm{B}-(\mathrm{A}-\mathrm{B}) *\left((1+((\mathrm{C} * \mathrm{X} 13) \wedge \mathrm{D}))^{\wedge}((\mathrm{E}-1) / \mathrm{D})\right)\right)^{*}(-1+((1+((\mathrm{C} * \mathrm{X} 13) \wedge \mathrm{D})) \wedge((\mathrm{E}-1) / \mathrm{D})))
$$$$
\mathrm{B} 14=\left(\mathrm{Y} 14-\mathrm{B}-(\mathrm{A}-\mathrm{B}) *\left(\left(1+\left(\left(\mathrm{C}^{*} \mathrm{X} 14\right) \wedge \mathrm{D}\right)\right)^{\wedge}((\mathrm{E}-1) / \mathrm{D})\right)\right)^{*}\left(-1+\left((1+((\mathrm{C} * \mathrm{X} 14) \wedge \mathrm{D}))^{\wedge}((\mathrm{E}-1) / \mathrm{D})\right)\right)
$$$$
\mathrm{B} 15=\left(\mathrm{Y} 15-\mathrm{B}-(\mathrm{A}-\mathrm{B})^{*}\left((1+((\mathrm{C} * \mathrm{X} 15) \wedge \mathrm{D}))^{\wedge}((\mathrm{E}-1) / \mathrm{D})\right)\right)^{*}\left(-1+\left((1+((\mathrm{C} * \mathrm{X} 15) \wedge \mathrm{D}))^{\wedge}((\mathrm{E}-1) / \mathrm{D})\right)\right)
$$$$
\mathrm{B} 16=\left(\mathrm{Y} 16-\mathrm{B}-(\mathrm{A}-\mathrm{B}) *\left(\left(1+\left(\left(\mathrm{C}^{*} \mathrm{X} 16\right) \wedge \mathrm{D}\right)\right)^{\wedge}((\mathrm{E}-1) / \mathrm{D})\right)\right)^{*}\left(-1+\left((1+((\mathrm{C} * \mathrm{X} 16) \wedge \mathrm{D}))^{\wedge}((\mathrm{E}-1) / \mathrm{D})\right)\right)
$$$$
\mathrm{B} 17=\left(\mathrm{Y} 17-\mathrm{B}-(\mathrm{A}-\mathrm{B})^{*}\left(\left(1+\left(\left(\mathrm{C}^{*} \mathrm{X} 17\right) \wedge \mathrm{D}\right)\right)^{\wedge}((\mathrm{E}-1) / \mathrm{D})\right)\right)^{*}\left(-1+\left((1+((\mathrm{C} * \mathrm{X} 17) \wedge \mathrm{D}))^{\wedge}((\mathrm{E}-1) / \mathrm{D})\right)\right)
$$$$
\mathrm{B} 18=\left(\mathrm{Y} 18-\mathrm{B}-(\mathrm{A}-\mathrm{B})^{*}\left((1+((\mathrm{C} * \mathrm{X} 18) \wedge \mathrm{D}))^{\wedge}((\mathrm{E}-1) / \mathrm{D})\right)\right)^{*}\left(-1+\left((1+((\mathrm{C} * \mathrm{X} 18) \wedge \mathrm{D}))^{\wedge}((\mathrm{E}-1) / \mathrm{D})\right)\right)
$$$$
\mathrm{B} 19=(\mathrm{Y} 19-\mathrm{B}-(\mathrm{A}-\mathrm{B}) *((1+((\mathrm{C} * \mathrm{X} 19) \wedge \mathrm{D})) \wedge((\mathrm{E}-1) / \mathrm{D})))^{*}(-1+((1+((\mathrm{C} * \mathrm{X} 19) \wedge \mathrm{D})) \wedge((\mathrm{E}-1) / \mathrm{D})))
$$

\# Expansión de cada uno de los términos de la derivada de la función respecto al parámetro C

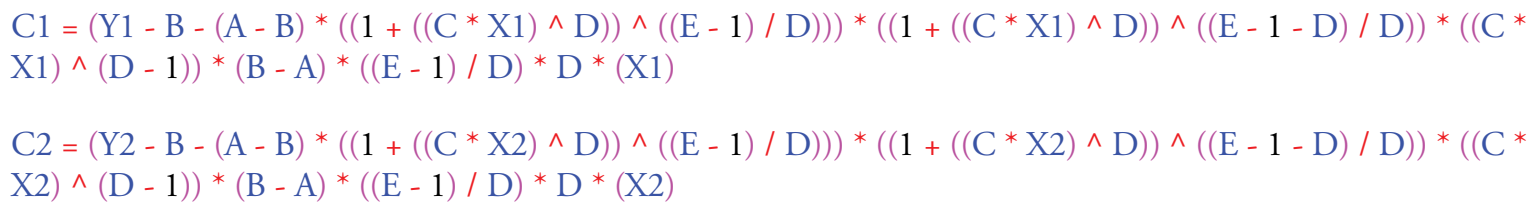




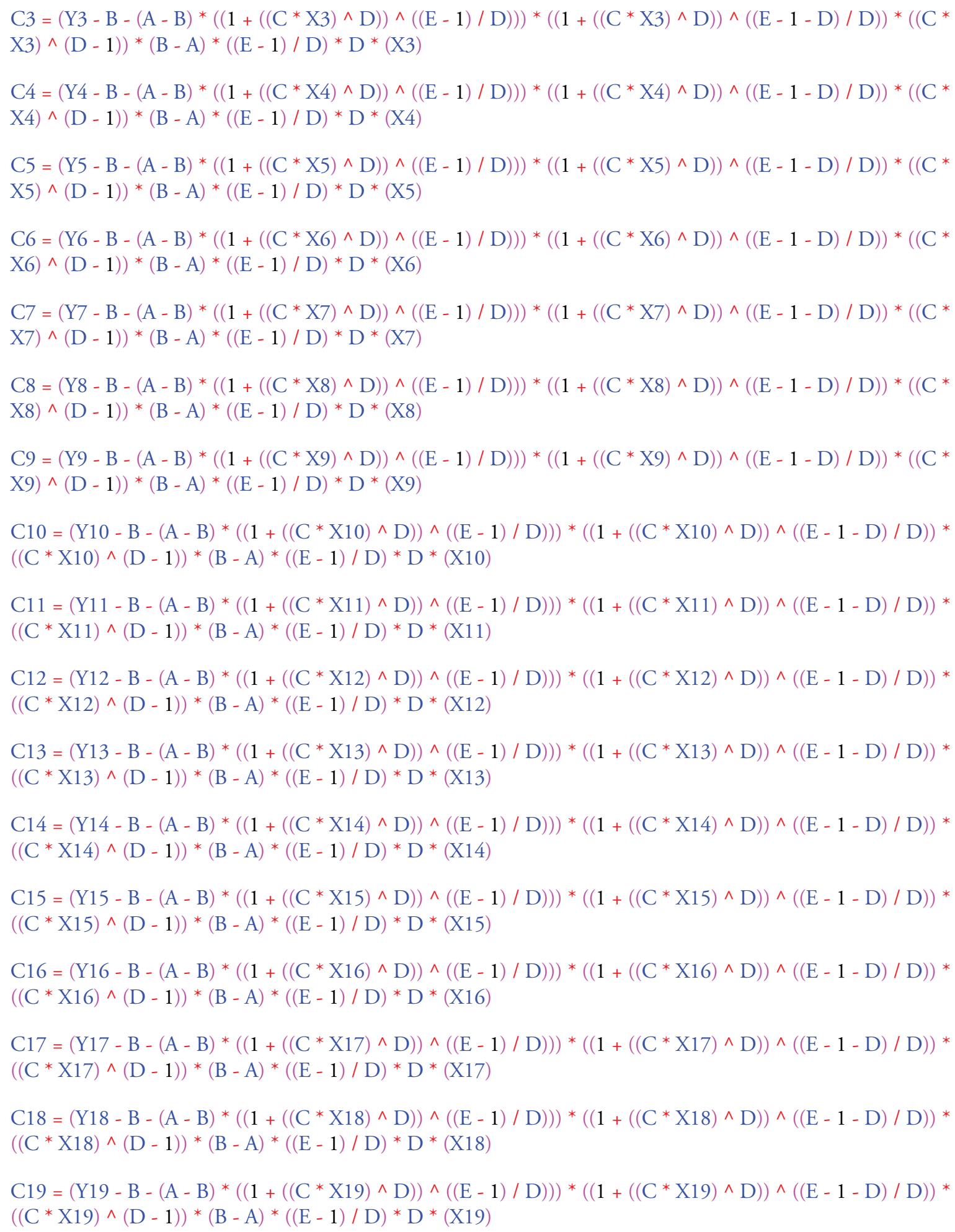




\begin{abstract}
$\mathrm{D} 3=\left(\mathrm{Y} 3-\mathrm{B}-(\mathrm{A}-\mathrm{B}) *\left(\left(1+\left((\mathrm{C} * \mathrm{X} 3)^{\wedge} \mathrm{D}\right)\right)^{\wedge}((\mathrm{E}-1) / \mathrm{D})\right)\right)^{*}((1+((\mathrm{C} * \mathrm{X} 3) \wedge \mathrm{D})) \wedge((\mathrm{E}-1) / \mathrm{D}))^{*}(\mathrm{~B}-\mathrm{A})^{*}$ $\left(((\mathrm{E}-1) / \mathrm{D}) *\left(\left(\left(\ln \left(\mathrm{C}^{*} \mathrm{X} 3\right)\right) *\left(\left(\mathrm{C}^{*} \mathrm{X} 3\right)^{\wedge} \mathrm{D}\right)\right) /\left(1+\left(\left(\mathrm{C}^{*} \mathrm{X} 3\right)^{\wedge} \mathrm{D}\right)\right)\right)-((\mathrm{E}-1) /(\mathrm{D} \wedge 2))^{*} \ln \left(1+\left(\left(\mathrm{C}^{*} \mathrm{X} 3\right)^{\wedge} \mathrm{D}\right)\right)\right)$ $\mathrm{D} 4=\left(\mathrm{Y} 4-\mathrm{B}-(\mathrm{A}-\mathrm{B}) *\left((1+((\mathrm{C} * \mathrm{X} 4) \wedge \mathrm{D}))^{\wedge}((\mathrm{E}-1) / \mathrm{D})\right)\right)^{*}((1+((\mathrm{C} * \mathrm{X} 4) \wedge \mathrm{D})) \wedge((\mathrm{E}-1) / \mathrm{D}))^{*}(\mathrm{~B}-\mathrm{A}) *$ $\left(((\mathrm{E}-1) / \mathrm{D}) *\left(\left(\left(\ln \left(\mathrm{C}^{*} \mathrm{X} 4\right)\right) *\left(\left(\mathrm{C}^{*} \mathrm{X} 4\right) \wedge \mathrm{D}\right)\right) /\left(1+\left(\left(\mathrm{C}^{*} \mathrm{X} 4\right)^{\wedge} \mathrm{D}\right)\right)\right)-((\mathrm{E}-1) /(\mathrm{D} \wedge 2)) * \ln (1+((\mathrm{C} * \mathrm{X} 4) \wedge \mathrm{D}))\right)$ $\mathrm{D} 5=\left(\mathrm{Y} 5-\mathrm{B}-(\mathrm{A}-\mathrm{B})^{*}\left(\left(1+\left((\mathrm{C} * \mathrm{X} 5)^{\wedge} \mathrm{D}\right)\right)^{\wedge}((\mathrm{E}-1) / \mathrm{D})\right)\right)^{*}((1+((\mathrm{C} * \mathrm{X} 5) \wedge \mathrm{D})) \wedge((\mathrm{E}-1) / \mathrm{D}))^{*}(\mathrm{~B}-\mathrm{A})^{*}$ $\left(((\mathrm{E}-1) / \mathrm{D}) *\left(\left(\left(\ln \left(\mathrm{C}^{*} \mathrm{X} 5\right)\right) *\left(\left(\mathrm{C}^{*} \mathrm{X} 5\right)^{\wedge} \mathrm{D}\right)\right) /\left(1+\left(\left(\mathrm{C}^{*} \mathrm{X} 5\right)^{\wedge} \mathrm{D}\right)\right)\right)-((\mathrm{E}-1) /(\mathrm{D} \wedge 2)) * \ln \left(1+\left((\mathrm{C} * \mathrm{X} 5)^{\wedge} \mathrm{D}\right)\right)\right)$ $\mathrm{D} 6=\left(\mathrm{Y} 6-\mathrm{B}-(\mathrm{A}-\mathrm{B}) *\left((1+((\mathrm{C} * \mathrm{X} 6) \wedge \mathrm{D}))^{\wedge}((\mathrm{E}-1) / \mathrm{D})\right)\right)^{*}((1+((\mathrm{C} * \mathrm{X} 6) \wedge \mathrm{D})) \wedge((\mathrm{E}-1) / \mathrm{D}))^{*}(\mathrm{~B}-\mathrm{A})^{*}$ $\left(((\mathrm{E}-1) / \mathrm{D}) *\left(\left(\left(\ln \left(\mathrm{C}^{*} \mathrm{X} 6\right)\right) *\left(\left(\mathrm{C}^{*} \mathrm{X} 6\right) \wedge \mathrm{D}\right)\right) /\left(1+\left(\left(\mathrm{C}^{*} \mathrm{X} 6\right)^{\wedge} \mathrm{D}\right)\right)\right)-((\mathrm{E}-1) /(\mathrm{D} \wedge 2)) * \ln \left(1+\left(\left(\mathrm{C}^{*} \mathrm{X} 6\right) \wedge \mathrm{D}\right)\right)\right)$ $\mathrm{D} 7=\left(\mathrm{Y} 7-\mathrm{B}-(\mathrm{A}-\mathrm{B}) *\left((1+((\mathrm{C} * \mathrm{X} 7) \wedge \mathrm{D}))^{\wedge}((\mathrm{E}-1) / \mathrm{D})\right)\right)^{*}\left((1+((\mathrm{C} * \mathrm{X} 7) \wedge \mathrm{D}))^{\wedge}((\mathrm{E}-1) / \mathrm{D})\right)^{*}(\mathrm{~B}-\mathrm{A})^{*}$ $\left(((\mathrm{E}-1) / \mathrm{D})^{*}\left(\left(\left(\ln \left(\mathrm{C}^{*} \mathrm{X} 7\right)\right) *\left(\left(\mathrm{C}^{*} \mathrm{X} 7\right) \wedge \mathrm{D}\right)\right) /\left(1+\left(\left(\mathrm{C}^{*} \mathrm{X} 7\right) \wedge \mathrm{D}\right)\right)\right)-((\mathrm{E}-1) /(\mathrm{D} \wedge 2))^{*} \ln \left(1+\left(\left(\mathrm{C}^{*} \mathrm{X} 7\right) \wedge \mathrm{D}\right)\right)\right)$ $\mathrm{D} 8=\left(\mathrm{Y} 8-\mathrm{B}-(\mathrm{A}-\mathrm{B}) *\left(\left(1+\left((\mathrm{C} * \mathrm{X} 8)^{\wedge} \mathrm{D}\right)\right)^{\wedge}((\mathrm{E}-1) / \mathrm{D})\right)\right)^{*}\left((1+((\mathrm{C} * \mathrm{X} 8) \wedge \mathrm{D}))^{\wedge}((\mathrm{E}-1) / \mathrm{D})\right)^{*}(\mathrm{~B}-\mathrm{A})^{*}$ $\left(((\mathrm{E}-1) / \mathrm{D}) *\left(\left(\left(\ln \left(\mathrm{C}^{*} \mathrm{X} 8\right)\right) *\left(\left(\mathrm{C}^{*} \mathrm{X} 8\right) \wedge \mathrm{D}\right)\right) /\left(1+\left(\left(\mathrm{C}^{*} \mathrm{X} 8\right)^{\wedge} \mathrm{D}\right)\right)\right)-((\mathrm{E}-1) /(\mathrm{D} \wedge 2)) * \ln \left(1+\left((\mathrm{C} * \mathrm{X} 8)^{\wedge} \mathrm{D}\right)\right)\right)$ $\mathrm{D} 9=\left(\mathrm{Y} 9-\mathrm{B}-(\mathrm{A}-\mathrm{B})^{*}\left((1+((\mathrm{C} * \mathrm{X} 9) \wedge \mathrm{D}))^{\wedge}((\mathrm{E}-1) / \mathrm{D})\right)\right)^{*}((1+((\mathrm{C} * \mathrm{X} 9) \wedge \mathrm{D})) \wedge((\mathrm{E}-1) / \mathrm{D})) *(\mathrm{~B}-\mathrm{A}) *$ $\left(((\mathrm{E}-1) / \mathrm{D}) *\left(\left(\left(\ln \left(\mathrm{C}^{*} \mathrm{X} 9\right)\right) *\left(\left(\mathrm{C}^{*} \mathrm{X} 9\right) \wedge \mathrm{D}\right)\right) /\left(1+\left(\left(\mathrm{C}^{*} \mathrm{X} 9\right)^{\wedge} \mathrm{D}\right)\right)\right)-((\mathrm{E}-1) /(\mathrm{D} \wedge 2)) * \ln \left(1+\left((\mathrm{C} * \mathrm{X} 9)^{\wedge} \mathrm{D}\right)\right)\right)$ $\mathrm{D} 10=(\mathrm{Y} 10-\mathrm{B}-(\mathrm{A}-\mathrm{B}) *((1+((\mathrm{C} * \mathrm{X} 10) \wedge \mathrm{D})) \wedge((\mathrm{E}-1) / \mathrm{D}))) *((1+((\mathrm{C} * \mathrm{X} 10) \wedge \mathrm{D})) \wedge((\mathrm{E}-1) / \mathrm{D})) *$ $(\mathrm{B}-\mathrm{A}) *\left(((\mathrm{E}-1) / \mathrm{D}) *\left(\left(\left(\ln \left(\mathrm{C}^{*} \mathrm{X} 10\right)\right) *\left(\left(\mathrm{C}^{*} \mathrm{X} 10\right) \wedge \mathrm{D}\right)\right) /(1+((\mathrm{C} * \mathrm{X} 10) \wedge \mathrm{D}))\right)-((\mathrm{E}-1) /(\mathrm{D} \wedge 2)) * \ln (1+\right.$ $\left.\left.\left(\left(\mathrm{C}^{*} \mathrm{X} 10\right) \wedge \mathrm{D}\right)\right)\right)$
\end{abstract}

$\mathrm{D} 11=\left(\mathrm{Y} 11-\mathrm{B}-(\mathrm{A}-\mathrm{B})^{*}\left((1+((\mathrm{C} * \mathrm{X} 11) \wedge \mathrm{D}))^{\wedge}((\mathrm{E}-1) / \mathrm{D})\right)\right)^{*}((1+((\mathrm{C} * \mathrm{X} 11) \wedge \mathrm{D})) \wedge((\mathrm{E}-1) / \mathrm{D}))^{*}$ $(\mathrm{B}-\mathrm{A}) *(((\mathrm{E}-1) / \mathrm{D}) *(((\ln (\mathrm{C} * \mathrm{X} 11)) *((\mathrm{C} * \mathrm{X} 11) \wedge \mathrm{D})) /(1+((\mathrm{C} * \mathrm{X} 11) \wedge \mathrm{D})))-((\mathrm{E}-1) /(\mathrm{D} \wedge 2)) * \ln (1+$ $\left.\left.\left(\left(\mathrm{C}^{*} \mathrm{X} 11\right) \wedge \mathrm{D}\right)\right)\right)$

$\mathrm{D} 12=\left(\mathrm{Y} 12-\mathrm{B}-(\mathrm{A}-\mathrm{B})^{*}\left((1+((\mathrm{C} * \mathrm{X} 12) \wedge \mathrm{D}))^{\wedge}((\mathrm{E}-1) / \mathrm{D})\right)\right)^{*}((1+((\mathrm{C} * \mathrm{X} 12) \wedge \mathrm{D})) \wedge((\mathrm{E}-1) / \mathrm{D}))^{*}$ $(\mathrm{B}-\mathrm{A}) *\left(((\mathrm{E}-1) / \mathrm{D}) *\left(\left(\left(\ln \left(\mathrm{C}^{*} \mathrm{X} 12\right)\right) *\left(\left(\mathrm{C}^{*} \mathrm{X} 12\right) \wedge \mathrm{D}\right)\right) /(1+((\mathrm{C} * \mathrm{X} 12) \wedge \mathrm{D}))\right)-((\mathrm{E}-1) /(\mathrm{D} \wedge 2)) * \ln (1+\right.$ $\left.\left.\left(\left(\mathrm{C}^{*} \mathrm{X} 12\right) \wedge \mathrm{D}\right)\right)\right)$

$\mathrm{D} 13=\left(\mathrm{Y} 13-\mathrm{B}-(\mathrm{A}-\mathrm{B})^{*}\left(\left(1+\left((\mathrm{C} * \mathrm{X} 13)^{\wedge} \mathrm{D}\right)\right)^{\wedge}((\mathrm{E}-1) / \mathrm{D})\right)\right)^{*}((1+((\mathrm{C} * \mathrm{X} 13) \wedge \mathrm{D})) \wedge((\mathrm{E}-1) / \mathrm{D}))^{*}$ $(\mathrm{B}-\mathrm{A}) *\left(((\mathrm{E}-1) / \mathrm{D}) *\left(\left(\left(\ln \left(\mathrm{C}^{*} \mathrm{X} 13\right)\right) *\left(\left(\mathrm{C}^{*} \mathrm{X} 13\right) \wedge \mathrm{D}\right)\right) /\left(1+\left(\left(\mathrm{C}^{*} \mathrm{X} 13\right) \wedge \mathrm{D}\right)\right)\right)-((\mathrm{E}-1) /(\mathrm{D} \wedge 2)) * \ln (1+\right.$ $\left.\left.\left(\left(\mathrm{C}^{*} \mathrm{X} 13\right) \wedge \mathrm{D}\right)\right)\right)$

$\mathrm{D} 14=\left(\mathrm{Y} 14-\mathrm{B}-(\mathrm{A}-\mathrm{B}) *\left((1+((\mathrm{C} * \mathrm{X} 14) \wedge \mathrm{D}))^{\wedge}((\mathrm{E}-1) / \mathrm{D})\right)\right)^{*}((1+((\mathrm{C} * \mathrm{X} 14) \wedge \mathrm{D})) \wedge((\mathrm{E}-1) / \mathrm{D})) *$ $(\mathrm{B}-\mathrm{A}) *\left(((\mathrm{E}-1) / \mathrm{D}) *\left(\left(\left(\ln \left(\mathrm{C}^{*} \mathrm{X} 14\right)\right) *\left(\left(\mathrm{C}^{*} \mathrm{X} 14\right) \wedge \mathrm{D}\right)\right) /(1+((\mathrm{C} * \mathrm{X} 14) \wedge \mathrm{D}))\right)-((\mathrm{E}-1) /(\mathrm{D} \wedge 2)) * \ln (1+\right.$ $\left.\left.\left(\left(\mathrm{C}^{*} \mathrm{X} 14\right) \wedge \mathrm{D}\right)\right)\right)$

$\mathrm{D} 15=\left(\mathrm{Y} 15-\mathrm{B}-(\mathrm{A}-\mathrm{B}) *\left((1+((\mathrm{C} * \mathrm{X} 15) \wedge \mathrm{D}))^{\wedge}((\mathrm{E}-1) / \mathrm{D})\right)\right)^{*}((1+((\mathrm{C} * \mathrm{X} 15) \wedge \mathrm{D})) \wedge((\mathrm{E}-1) / \mathrm{D})) *$ $(\mathrm{B}-\mathrm{A}) *\left(((\mathrm{E}-1) / \mathrm{D}) *\left(\left(\left(\ln \left(\mathrm{C}^{*} \mathrm{X} 15\right)\right) *((\mathrm{C} * \mathrm{X} 15) \wedge \mathrm{D})\right) /(1+((\mathrm{C} * \mathrm{X} 15) \wedge \mathrm{D}))\right)-((\mathrm{E}-1) /(\mathrm{D} \wedge 2)) * \ln (1+\right.$ $\left.\left.\left(\left(\mathrm{C}^{*} \mathrm{X} 15\right) \wedge \mathrm{D}\right)\right)\right)$

$\mathrm{D} 16=\left(\mathrm{Y} 16-\mathrm{B}-(\mathrm{A}-\mathrm{B})^{*}\left((1+((\mathrm{C} * \mathrm{X} 16) \wedge \mathrm{D}))^{\wedge}((\mathrm{E}-1) / \mathrm{D})\right)\right)^{*}((1+((\mathrm{C} * \mathrm{X} 16) \wedge \mathrm{D})) \wedge((\mathrm{E}-1) / \mathrm{D}))^{*}$ $(\mathrm{B}-\mathrm{A}) *(((\mathrm{E}-1) / \mathrm{D}) *(((\ln (\mathrm{C} * \mathrm{X} 16)) *((\mathrm{C} * \mathrm{X} 16) \wedge \mathrm{D})) /(1+((\mathrm{C} * \mathrm{X} 16) \wedge \mathrm{D})))-((\mathrm{E}-1) /(\mathrm{D} \wedge 2)) * \ln (1+$ $\left.\left.\left(\left(\mathrm{C}^{*} \mathrm{X} 16\right) \wedge \mathrm{D}\right)\right)\right)$

$\mathrm{D} 17=\left(\mathrm{Y} 17-\mathrm{B}-(\mathrm{A}-\mathrm{B})^{*}\left((1+((\mathrm{C} * \mathrm{X} 17) \wedge \mathrm{D}))^{\wedge}((\mathrm{E}-1) / \mathrm{D})\right)\right)^{*}((1+((\mathrm{C} * \mathrm{X} 17) \wedge \mathrm{D})) \wedge((\mathrm{E}-1) / \mathrm{D}))^{*}$ $(\mathrm{B}-\mathrm{A}) *\left(((\mathrm{E}-1) / \mathrm{D}) *\left(\left(\left(\ln \left(\mathrm{C}^{*} \mathrm{X} 17\right)\right) *\left(\left(\mathrm{C}^{*} \mathrm{X} 17\right) \wedge \mathrm{D}\right)\right) /(1+((\mathrm{C} * \mathrm{X} 17) \wedge \mathrm{D}))\right)-((\mathrm{E}-1) /(\mathrm{D} \wedge 2)) * \ln (1+\right.$ $((\mathrm{C} * \mathrm{X} 17) \wedge \mathrm{D})))$

$\mathrm{D} 18=(\mathrm{Y} 18-\mathrm{B}-(\mathrm{A}-\mathrm{B}) *((1+((\mathrm{C} * \mathrm{X} 18) \wedge \mathrm{D})) \wedge((\mathrm{E}-1) / \mathrm{D})))^{*}((1+((\mathrm{C} * \mathrm{X} 18) \wedge \mathrm{D})) \wedge((\mathrm{E}-1) / \mathrm{D})) *$ $(\mathrm{B}-\mathrm{A}) *\left(((\mathrm{E}-1) / \mathrm{D}) *\left(\left(\left(\ln \left(\mathrm{C}^{*} \mathrm{X} 18\right)\right) *((\mathrm{C} * \mathrm{X} 18) \wedge \mathrm{D})\right) /(1+((\mathrm{C} * \mathrm{X} 18) \wedge \mathrm{D}))\right)-((\mathrm{E}-1) /(\mathrm{D} \wedge 2)) * \ln (1+\right.$ $\left.\left.\left(\left(\mathrm{C}^{*} \mathrm{X} 18\right) \wedge \mathrm{D}\right)\right)\right)$

$\mathrm{D} 19=\left(\mathrm{Y} 19-\mathrm{B}-(\mathrm{A}-\mathrm{B}) *\left((1+((\mathrm{C} * \mathrm{X} 19) \wedge \mathrm{D}))^{\wedge}((\mathrm{E}-1) / \mathrm{D})\right)\right)^{*}((1+((\mathrm{C} * \mathrm{X} 19) \wedge \mathrm{D})) \wedge((\mathrm{E}-1) / \mathrm{D})) *$ $(\mathrm{B}-\mathrm{A}) *(((\mathrm{E}-1) / \mathrm{D}) *(((\ln (\mathrm{C} * \mathrm{X} 19)) *((\mathrm{C} * \mathrm{X} 19) \wedge \mathrm{D})) /(1+((\mathrm{C} * \mathrm{X} 19) \wedge \mathrm{D})))-((\mathrm{E}-1) /(\mathrm{D} \wedge 2)) * \ln (1+$ $\left.\left.\left(\left(\mathrm{C}^{*} \mathrm{X} 19\right) \wedge \mathrm{D}\right)\right)\right)$ 
\# Expansión de cada uno de los términos de la derivada de la función respecto al parámetro E

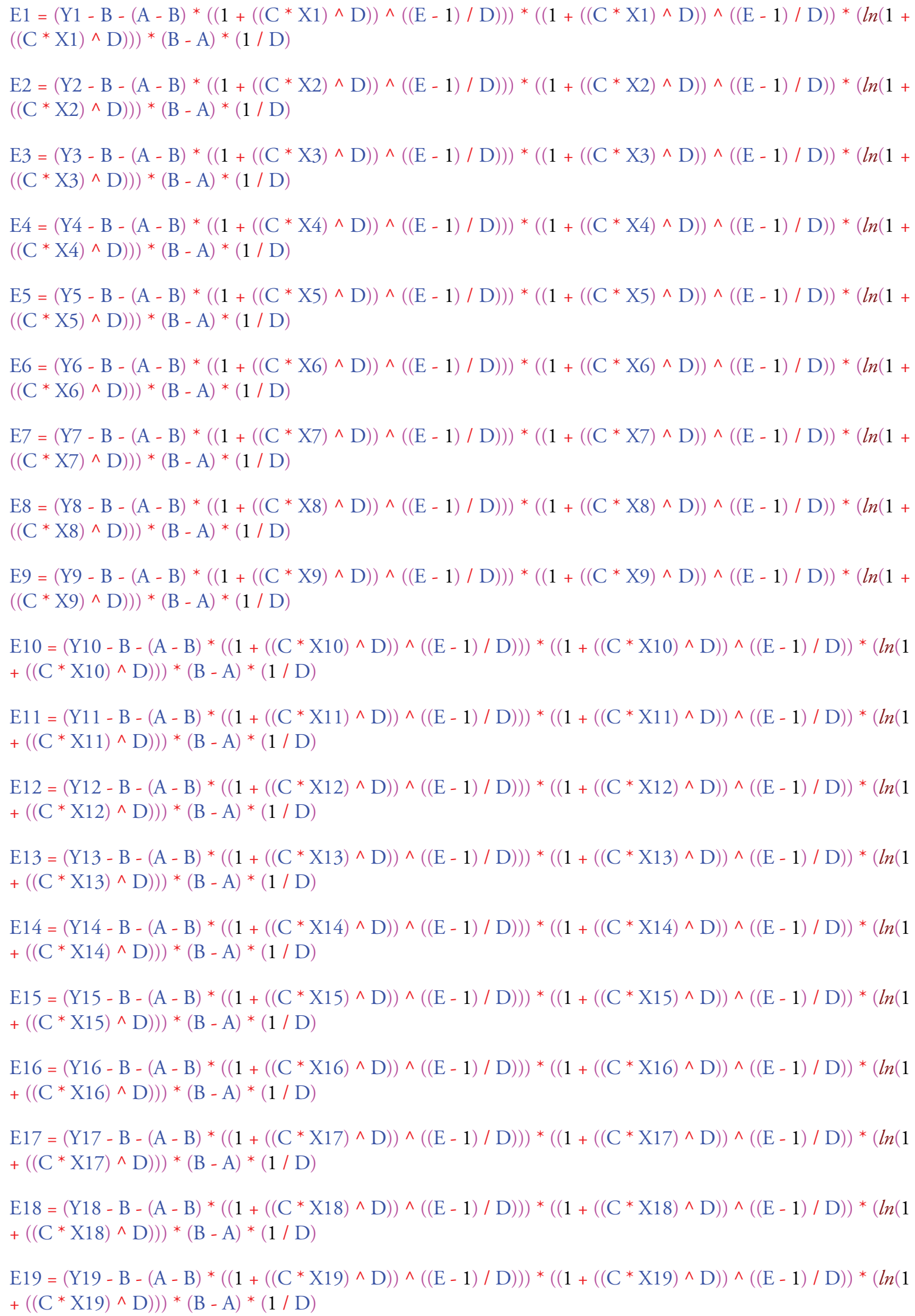




$\begin{array}{ll}\text { Valores de la velocidad de corte } & \text { Valores de la viscosidad aparente } \\ \mathrm{X} 1=0.624 & \mathrm{Y} 1=37.211 \\ \mathrm{X} 2=0.700 & \mathrm{Y} 2=38.771 \\ \mathrm{X} 3=0.792 & \mathrm{Y} 3=39.229 \\ \mathrm{X} 4=1.17 & \mathrm{Y} 4=36.623 \\ \mathrm{X} 5=1.31 & \mathrm{Y} 5=37.198 \\ \mathrm{X} 6=1.70 & \mathrm{Y} 6=36.747 \\ \mathrm{X} 7=2.26 & \mathrm{Y} 7=34.592 \\ \mathrm{X} 8=2.70 & \mathrm{Y} 8=33.310 \\ \mathrm{X} 9=3.77 & \mathrm{Y} 9=28.02 \\ \mathrm{X} 10=4.61 & \mathrm{Y} 10=23.77 \\ \mathrm{X} 11=8.92 & \mathrm{Y} 11=12.72 \\ \mathrm{X} 12=23.2 & \mathrm{Y} 12=5.23 \\ \mathrm{X} 13=24.7 & \mathrm{Y} 13=5.07 \\ \mathrm{X} 14=41.35 & \mathrm{Y} 14=3.17 \\ \mathrm{X} 15=83.49 & \mathrm{Y} 15=1.64 \\ \mathrm{X} 16=97.19 & \mathrm{Y} 16=1.47 \\ \mathrm{X} 17=99.97 & \mathrm{Y} 17=1.42 \\ \mathrm{X} 18=136.07 & \mathrm{Y} 18=1.10 \\ \mathrm{X} 19=138.75 & \mathrm{Y} 19=1.08 \\ & \end{array}$


